\title{
Single-RF and Twin-RF Spatial Modulation for an Arbitrary Number of Transmit Antennas
}

\author{
Lixia Xiao, Yue Xiao, Member, IEEE, Longfei You, Ping Yang, Member, IEEE, Shaoqian Li, Fellow, \\ IEEE, and Lajos Hanzo, Fellow, IEEE
}

\begin{abstract}
Spatial Modulation (SM) constitutes an appealing low-complexity single Radio Frequency (RF)-aided Multiple-Input Multiple-Output (MIMO) technique. Conventional SM schemes tend to rely on transmit antenna (TA) configurations, where the number of TAs is a power of two. In order to circumvent this limitation, a novel single RF-aided SM conceived for an Arbitrary Number of TAs (SM-ATA) is proposed, which is then further developed to a twin-RF Enhanced SM-ATA (ESM-ATA) schedule for exploiting the diversity advantage of Space-Time Block Coding (STBC). Furthermore, low-complexity near-optimal detectors are designed for both the SM-ATA and ESM-ATA schemes. Our simulation results show that the proposed SM-ATA schemes offer almost the same performance as conventional SM systems, despite using a reduced number of transmit antennas at the same transmission rate. The proposed twin-RF ESM-ATA schemes provide a beneficial performance gain over the existing twinRF multiplexing based and space-time coding based SM schemes. Finally, an upper bound is derived for the Average Bit Error Probability (ABEP), which is confirmed by our simulation results.
\end{abstract}

Index Terms - Spatial Modulation (SM), MultipleInput Multiple-Output (MIMO), arbitrary transmit antennas, Space Time Block Coding (STBC).

\section{INTRODUCTION}

$\mathbf{S}$ PATIAL Modulation (SM) [1]-[6] constitutes a novel Multi-Input Multi-Output (MIMO) transmission technique, which exploits the activated antenna indices as an additional means of conveying information. Specifically, in the SM scheme, in each time instant, only a single Transmit Antenna (TA) is activated for transmitting the classic Amplitude and Phase Modulation (APM) symbols so as to avoid inter-antenna synchronization, while mitigating the inter-antenna inference. Furthermore, SM has been shown to be a promising large-scale MIMO technique for millimeter-wave communications [7]-[8].

L. Xiao, Y. Xiao, L. You, P. Yang, S. Li are with the National Key Laboratory of Science and Technology on Communications, University of Electronic Science and Technology of China 611731, Sichuan, China.

L. Xiao thanks the National Science Foundation of China under Grant number 61671131 and the Fundamental Research Funds For the Central Universities (NO. ZYGX2015J011).

L. Hanzo is with the school of Electronics and Computer Science, University of Southampton, Southampton SO17 1BJ, U.K. (email: lh@ecs.soton.ac.uk).

L. Hanzo would also like to gratefully acknowledge the financial support of the European Research Council's Advanced Fellow Grant. (C)IEEE TVT 2018
For the conventional single-RF SM scheme, $\log _{2}\left(N_{t}\right)$ bits are utilized to select the activated TA index from the index group of $\Lambda=\left\{1,2, \ldots, N_{t}\right\}$ and $\log _{2}(M)$ bits are mapped to an $M$-ary APM symbol, where $N_{t}$ is the number of TAs. As a result, the conventional SM is only applicable to specific TA configurations associated with $\log _{2}\left(N_{t}\right)$ being an integer. Recently, the issues of spatial constellation optimization, hybrid bit-to-symbol mapping, link adaptation, and constellation randomization were investigated in [9]-[16], which were applied to single-RF SM. However, the above-mentioned refined transceiver designs still remain unsuitable for an arbitrary number of TAs associated with a single RF.

In order to relax the integer power of two constraint imposed on the number of TAs, single-RF SM was designed for an arbitrary number of TAs in [17]-[20]. Specifically, the Fractional Bit Encoded Spatial Modulation (FBE-SM) scheme of [17] mapped the information bits by a block of base- $N_{t}$ representation numbers, but this technique may suffer from error propagation during block-based transmission. In order to improve the performance of the FBESM scheme, the Variable Throughput Modulation $(\mathrm{VTM})^{1}$ based bit-padding methods were developed in [18]-[19], which transmitted a variable number of information bits over the different activated TA indices. In [20], the authors proposed a 3-D mapping aided SM scheme, which relies on a total of $N_{t}$ constellation sets for $N_{t}$ activated indices. Naturally, the optimization of the $N_{t}$ constellation sets may impose extra complexity, especially for a large number of TAs and for a high transmission rate. Furthermore, the activated TA index of the schemes in [19]-[20] did not convey a fixed number of bits, which may impose some extra complexity during the bit-to-symbol mapping. As a result, developing a low-complexity bit-to-symbol mapping aided single-RF SM scheme for an arbitrary number of TAs, while retaining all the advantages of the conventional SM scheme remains an open question to be investigated.

Additionally, improved variants of multiple-RF aided SM were developed in [21]-[35] to further investigate the multiplexing [21]-[29] and diversity gains [30]-[35] of SM systems. For the multiplexing based SM schemes, the beam-forming based generalized SM scheme of [21] and the time-variant number of activated TAs based SM scheme of [28] employed $N_{t} \mathrm{RF}$ chains at the transmitter. The joint

\footnotetext{
${ }^{1}$ In [19] the terminology of Modulation-Varying (MV) was used for
} the VTM of this treatise. 
Spatial and Symbol Alphabet based SM (SSA-SM) scheme of [29] relied on $N_{t} / 2-\mathrm{RF}$ chains at the transmitter. The twin-RF based Quadrature SM (QSM) schemes of [25] and the Enhanced SM (ESM) scheme of [26]-[27] were suitable for the special case, when the number of TAs is an integer power of two. Only the Generalized SM (GSM) scheme of [22]-[24] can be applied for an arbitrary number of TAs relying on a twin-RF at the transmitter.

For the space time coding based SM schemes of [30]-[35], the double space-time transmit diversity based SM scheme of [34] and the spatially modulated orthogonal space-time block codes scheme of [35] employed at least four RF chains at the transmitter. The Space Time Block Coding SM (STBC-SM) scheme of [30], the high rate STBC-SM of [31], the high rate Multi-Strata Space-Time Coded SM (MSSTC-SM) scheme of [32] and the Modified Codeword based STBC-SM (MC-STBC-SM) of [33] employed twin$\mathrm{RF}$ chains at the transmitter. The schemes in [31]-[33] employed similar transmit antenna combination (TAC) groups to that of [30] and achieved a diversity gain by setting different amplitudes and phase rotations for the different TAC groups. As $N_{t}$ increases, the number of different TAC groups increases, hence they require more amplitudes and phases to distinguish the TAC groups. Consequently, the diversity gains of these schemes decrease as $N_{t}$ increases.

Against the above background, the contributions of this paper are summarized as follows:

1) A novel single RF-aided SM scheme is proposed for an Arbitrary Number of TAs (SM-ATA). Specifically, for any non-integer number of bits, the TA index group $\Lambda=\left\{1,2, \ldots, N_{t}\right\}$ is extended to an integer number of bits based index group as $\Lambda_{p}=$ $\left\{1,2, \ldots, N_{t}, q_{1} e^{j \theta}, \ldots, q_{L-N_{t}} e^{j \theta}\right\} q_{i}=\left(1,2, \ldots, N_{t}\right), i=$ $\left(1, \ldots, L-N_{t}\right)$, where $L=2^{\left\lfloor\log _{2}\left(N_{t}\right)\right\rfloor+1}$ is the size of $\Lambda_{p}$ and $\downarrow \cdot \mid$ is the floor operator. Explicitly, when the element $q_{i} e^{j \theta}$ is selected in the above SM-ATA mapping, a phase rotation is applied to the classic $M$-ary constellation symbols transmitted by the $q_{i}$ th TA.

2) Then, based on the above SM-ATA scheme, a twinRF Enhanced SM-ATA (ESM-ATA) arrangement is proposed, whose TAs are divided into two sets. The above-mentioned SM-ATA scheme relies on $N(N<$ $\left.N_{t}\right)$ TAs, while the remaining $\left(N_{t}-N\right)$ TAs are used for enhancing the integrity of the modulated SMATA symbols by invoking Alamouti's Space Time Block Coding (STBC).

3) Furthermore, an upper bound of the Average Bit Error Probability (ABEP) is derived for the proposed SM-ATA and ESM-ATA schemes. Finally, lowcomplexity detectors are developed for both the SMATA and ESM-ATA schemes. Our simulation results demonstrate that the proposed SM-ATA scheme approaches the performance of its conventional SM counterpart at the same throughput, despite having a reduced number of TAs. Finally, the further evolved ESM-ATA scheme is capable of providing a beneficial performance gain over the existing twinRF SM schemes at the same throughput and at the same number of TAs.

The remainder of this paper is organized as follows. Section II gives a rudimentary introduction to the conventional SM system. In Section III, the proposed single-RF SM-ATA is introduced and a low-complexity ML detector is developed. In Section IV, we propose the twin-RF ESMATA scheme and its low-complexity near-optimal detector. Our theoretical analysis is presented in Section V, while Section VI presents our simulation results. Finally, Section VII concludes this paper.

Notation: $\|\cdot\|_{F}$ denotes the Frobenious norm of a matrix; $|\cdot|$ represents the magnitude of a complex quantity; $(\cdot)^{T}$ and $(\cdot)^{H}$ stand for the transpose and the Hermitian transpose of a vector/matrix, respectively.

\section{Conventional SM System}

We consider a SM system having $N_{t}$ TAs and $N_{r}$ receive antennas communicating over flat Rayleigh fading channels. In the conventional SM scheme, the information bits are partitioned into two parts, whose $\log _{2}\left(N_{t}\right)$ bits are used to select an active TA index vector from the index vector set $\Omega_{c}=\left\{\mathbf{e}_{1}, \mathbf{e}_{2}, \ldots, \mathbf{e}_{N_{t}}\right\}$, where $\mathbf{e}_{q} 1 \leq q \leq N_{t}$ is selected from the $N_{t}$-dimensional standard basis vectors (i.e. $\mathbf{e}_{1}=[1,0, \ldots, 0]^{T}$ ) and $\log _{2}(M)$ bits are mapped to an $M$-ary APM symbol $s$. Based on the above mapping rule, the transmitted signal $\mathbf{x} \in \mathbb{C}^{N_{t} \times 1}$ can be represented as

$$
\mathbf{x}=\mathbf{e}_{q} s=[\underbrace{0, \ldots, 0}_{q-1}, s, \underbrace{0, \ldots, 0}_{N_{t}-q}]^{T},
$$

where $q \in \Lambda$ denotes the index of the activated TA.

For the conventional SM system, when $\log _{2}\left(N_{t}\right)$ is not an integer, conventional SM mapping becomes impractical. As a result, conventional SM is only suitable, when the number of TAs is a power of two. In order to address this issue, an improved SM scheme is proposed for an arbitrary number of TAs in next section.

\section{Single-RF Spatial Modulation for An Arbitrary Number of Transmit Antennas}

In this section, single-RF $\mathrm{SM}$ is proposed for an arbitrary number of TAs. Specifically, when the value of $\log _{2}\left(N_{t}\right)$ is not an integer, we extend the index group $\Lambda=\left\{1,2, \ldots, N_{t}\right\}$ to an index group having an integer number of bits as $\Lambda_{p}=\left\{1,2, \ldots, N_{t}, q_{1} e^{j \theta}, \ldots, q_{L-N_{t}} e^{j \theta}\right\} q_{i}=$ $\left(1,2, \ldots, N_{t}\right), i=\left(1, \ldots, L-N_{t}\right)$, where $L=2^{\left\lfloor\log _{2}\left(N_{t}\right)\right\rfloor+1}$ is the size of $\Lambda_{p}$. Explicitly, the element $q_{i} e^{j \theta}$ in the above SM-ATA mapping represents a phase rotation, which is applied to the classic $M$-ary constellation symbols transmitted by the $q_{i}$-th TA.

Example $N_{t}=5$ : For the case of $N_{t}=5$, the conventional index group $\Lambda=\{1,2,3,4,5\}$ is unsuitable for index mapping. For simplicity, we can extend the index group as $\Lambda_{p}=\left\{1,2,3,4,5,1 e^{j \theta}, 2 e^{j \theta}, 3 e^{j \theta}\right\}$. As a result, for the 


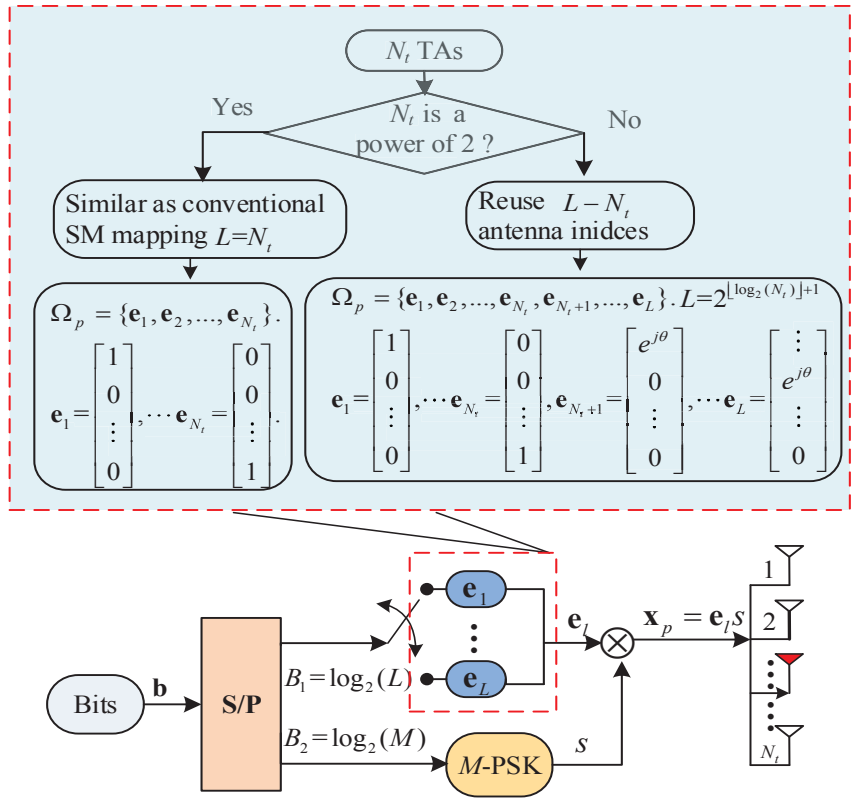

Fig. 1. System model of the proposed SM-ATA scheme.

BPSK-aided SM with $N_{t}=5$, the SM-ATA symbol set can be expressed as

$$
\begin{array}{cccccccccc}
\mathbb{X}_{\text {SM-ATA }}^{5}=\left[\begin{array}{cccccccc}
1 & 0 & 0 & 0 & 0 & 1 e^{j \theta} & 0 & 0 \\
0 & 1 & 0 & 0 & 0 & 0 & 1 e^{j \theta} & 0 \\
0 & 0 & 1 & 0 & 0 & 0 & 0 & 1 e^{j \theta} \\
0 & 0 & 0 & 1 & 0 & 0 & 0 & 0 \\
0 & 0 & 0 & 0 & 1 & 0 & 0 & 0 \\
-1 & 0 & 0 & 0 & 0 & -1 e^{j \theta} & 0 & 0 \\
0 & -1 & 0 & 0 & 0 & 0 & -1 e^{j \theta} & 0 \\
0 & 0 & -1 & 0 & 0 & 0 & 0 & -1 e^{j \theta} \\
0 & 0 & 0 & -1 & 0 & 0 & 0 & 0 \\
0 & 0 & 0 & 0 & -1 & 0 & 0 & 0
\end{array}\right] .
\end{array}
$$

Furthermore, the bit-to-symbol mapping process of the

\begin{tabular}{|c|c|c|c|c|}
\hline Scheme & $\begin{array}{c}\begin{array}{c}\text { Input } \\
\text { bits }\end{array} \\
\end{array}$ & $\begin{array}{c}\text { Mapping } \\
\text { Index } l\end{array}$ & $\begin{array}{c}\text { Activated } \\
\text { TA Index } q\end{array}$ & $\begin{array}{c}\text { SM-ATA } \\
\text { Symbol }\end{array}$ \\
\hline \multirow{16}{*}{$N_{t}=5$} & 0000 & 1 & 1 & {$\left[\begin{array}{lllll}-1 & 0 & 0 & 0 & 0\end{array}\right]^{T}$} \\
\hline & 0001 & 1 & 1 & {$\left[\begin{array}{lllll}1 & 0 & 0 & 0 & 0\end{array}\right]^{T}$} \\
\hline & 0010 & 2 & 2 & {$\left[\begin{array}{lllll}0 & -1 & 0 & 0 & 0\end{array}\right]^{T}$} \\
\hline & 0011 & 2 & 2 & {$\left[\begin{array}{lllll}0 & 1 & 0 & 0 & 0\end{array}\right]^{T}$} \\
\hline & 0100 & 3 & 3 & {$\left[\begin{array}{lllll}0 & 0 & -1 & 0 & 0\end{array}\right]^{T}$} \\
\hline & 0101 & 3 & 3 & {$\left[\begin{array}{lllll}0 & 0 & 1 & 0 & 0\end{array}\right]^{T}$} \\
\hline & 0110 & 4 & 4 & {$\left[\begin{array}{lllll}0 & 0 & 0 & -1 & 0\end{array}\right]^{T}$} \\
\hline & 0111 & 4 & 4 & {$\left[\begin{array}{llllll}0 & 0 & 0 & 1 & 0\end{array}\right]^{T}$} \\
\hline & 1000 & 5 & 5 & {$\left[\begin{array}{lllll}0 & 0 & 0 & 0 & -1\end{array}\right]^{T}$} \\
\hline & 1001 & 5 & 5 & {$\left[\begin{array}{lllll}0 & 0 & 0 & 0 & 1\end{array}\right]^{T}$} \\
\hline & 1010 & 6 & 1 & {$\left[\begin{array}{lllll}-1 e^{j \theta} & 0 & 0 & 0 & 0\end{array}\right]^{T}$} \\
\hline & 1011 & 6 & 1 & {$\left[\begin{array}{lllll}1 e^{j \theta} & 0 & 0 & 0 & 0\end{array}\right]^{T}$} \\
\hline & 1100 & 7 & 2 & {$\left[\begin{array}{llllll}0 & -1 e^{j \theta} & 0 & 0 & 0\end{array}\right]^{T}$} \\
\hline & 1101 & 7 & 2 & {$\left[\begin{array}{lllllll}0 & 1 e^{j \theta} & 0 & 0 & 0 & 0\end{array}\right]^{T}$} \\
\hline & 1110 & 8 & 3 & {$\left[\begin{array}{lllll}0 & 0 & -1 e^{j \theta} & 0 & 0\end{array}\right]^{T}$} \\
\hline & 1111 & 8 & 3 & {$\left[\begin{array}{llllll}0 & 0 & 1 e^{j \theta} & 0 & 0 & 0\end{array}\right]^{T}$} \\
\hline
\end{tabular}
SM-ATA schemes of Example is presented in Table I.

TABLE I

BIT-TO-Symbol MAPPING FOR The BPSK-AIDED SM-ATA SCHEMES WITH $N_{t}=5$.

\section{A. The Generalized SM-ATA Design}

The generalized system model of the proposed SM-ATA scheme is shown in Fig. 1, which operates as follows.

Step 1: Determine the index vector set $\Omega_{p}$.

1) Determine whether the value of $\log _{2}\left(N_{t}\right)$ is an integer or not, i.e. wether $N_{t}$ is an integer power of two.

2) If $\log _{2}\left(N_{t}\right)$ is an integer, the index vector set is $\Omega_{p}=$ $\Omega_{c}$.

3) If $\log _{2}\left(N_{t}\right)$ is not an integer, we first round up $\log _{2}\left(N_{t}\right)$ to $\left\lceil\log _{2}\left(N_{t}\right)\right\rceil$, which is an integer number of bits, corresponding to an increased index group of $L=2^{\left\lceil\log _{2}\left(N_{t}\right)\right\rceil}$, despite only having $N_{t}$ TAs.

4) Select $\left(L-N_{t}\right)$ elements $\Lambda_{q}=\left\{q_{1}, \ldots, q_{L-N_{t}}\right\}$ from $\Lambda=\left\{1,2, \ldots, N_{t}\right\}$ based on certain specific rules. For example, we can assume that $q_{i}=i i=\left(1, \ldots, L-N_{t}\right)$ for simplicity.

5) Formulate the extended index group as $\Lambda_{p}=$ $\left\{\Lambda, \Lambda_{q} e^{j \theta\}}=\left\{1, \ldots, N_{t}, q_{1} e^{j \theta}, \ldots, q_{L-N_{t}} e^{j \theta}\right\}\right.$. Based on the $\Lambda_{p}$ obtained, the index vector set is formulated as $\Omega_{p}=\left\{\mathbf{e}_{1}, \ldots, \mathbf{e}_{N_{t}}, \mathbf{e}_{N_{t}+1}, \ldots, \mathbf{e}_{L}\right\}$, where we have

$$
\mathbf{e}_{l}=\left\{\begin{array}{l}
{[\underbrace{0, \ldots, 0}_{l-1}, 1, \underbrace{0 \ldots, 0}_{N_{t}-l}]^{T}, \text { if } 1 \leq l \leq N_{t}} \\
{[\underbrace{0, \ldots, 0}_{q_{l-N_{t}}-1}, e^{j \theta}, \underbrace{0 \ldots, 0}_{N_{t}-q_{l-N_{t}}}]^{T}, \text { if } N_{t}<l \leq L .}
\end{array} .\right.
$$

Step 2: SM-ATA mapping. Specifically, a block of $B=\left(B_{1}+B_{2}\right)$ information bits is partitioned into two regiments: 1) $B_{1}=\log _{2}(L)$ bits are utilized to select one of the elements from the set $\Omega_{p}$; and 2) $B_{2}=\log _{2}(M)$ bits are mapped to an $M$-ary APM symbol. Specifically, when the element $\mathbf{e}_{l}\left(l>N_{t}\right)$ is chosen, the phase of the $M$-ary APM symbol transmitted by the $\left(q_{l-N_{t}}\right)$-th TA is rotated by $\theta$. Consequently, the SM-ATA symbol $\mathbf{x}_{p} \in \mathbb{C}^{N_{t} \times 1}$ can be represented as

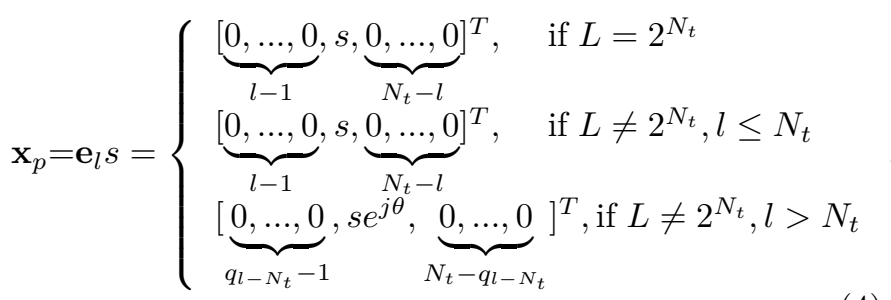

where $l$ denotes the mapping index, which is only the same as the activated TA index $q$ for the specific case of $l \leq N_{t}$. For a specific extended index group $\Lambda_{p}=\left\{\Lambda, \Lambda_{q} e^{\overline{j \theta}}\right\}=$ $\left\{1, \ldots, N_{t}, q_{1} e^{j \theta}, \ldots, q_{L-N_{t}} e^{j \theta}\right\}$, the relationship between $l$ and $q$ is expressed as

$$
\Lambda_{p}=\{\underbrace{1}_{l=q=1}, \ldots, \underbrace{N_{t}}_{l=q=N_{t}}, \underbrace{q_{1} e^{j \theta}}_{l=N_{t}+1, q=q_{1}}, \ldots, \underbrace{q_{L-N_{t} e^{j \theta}}}_{l=L, q=q_{L-N_{t}}}\} .
$$

\section{B. Optimization of single-RF aided SM-ATA}

Let us denote the transmit and receive signal of SMATA by $\mathbf{x}_{i}$ and $\mathbf{x}_{j}$, respectively. The distance between $\mathbf{x}_{i}$ 


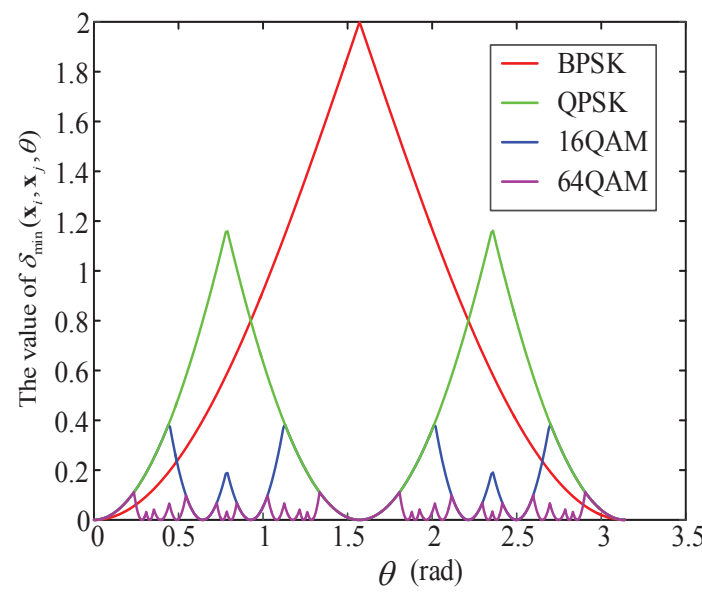

Fig. 2. The values of $\delta_{\min }\left(\mathbf{x}_{i}, \mathbf{x}_{j}, \theta\right)$ for different modulation orders.

and $\mathbf{x}_{j}$ is defined by

$$
\Delta_{s}^{H} \Delta_{\mathrm{s}}=\left\{\begin{array}{l}
\left(s_{i}-s_{j} e^{j \theta}\right)^{2}, \text { if } q_{i}=q_{j}, l_{i} \leq N_{t}, l_{j}>N_{t} \\
\left(s_{i} e^{j \theta}-s_{j}\right)^{2}, \text { if } q_{i}=q_{j}, l_{i}>N_{t}, l_{j} \leq N_{t} \\
\left|s_{i}\right|^{2}+\left|s_{j}\right|^{2}, \text { if } q_{i} \neq q_{j} \\
\left(s_{i}-s_{j}\right)^{2}, \text { Otherwise }
\end{array}\right.
$$

where $\Delta_{s}=\mathbf{x}_{i}-\mathbf{x}_{j}, q_{i}, q_{j}$ are the activated TA indices of $\mathbf{x}_{i}$ and $\mathbf{x}_{j}$, and $l_{i}, l_{j}$ are the corresponding mapping indices. The Minimum Distance (MD) between $\mathbf{x}_{i}$ and $\mathbf{x}_{j}$ associated with $\theta$ can be expressed as

$\delta_{\min }\left(\mathbf{x}_{i}, \mathbf{x}_{j}, \theta\right)=\left\{\begin{array}{l}\left(s_{i}-s_{j} e^{j \theta}\right)^{2}, \text { if } q_{i}=q_{j}, l_{i} \leq N_{t}, l_{j}>N_{t} \\ \left(s_{i} e^{j \theta}-s_{j}\right)^{2}, \text { if } q_{i}=q_{j}, l_{i}>N_{t}, l_{j} \leq N_{t}\end{array}\right.$

As a result, $\theta$ can be optimized by maximizing the MD as

$$
\hat{\theta}=\arg \max _{\theta}\left[\delta_{\min }\left(\mathbf{x}_{i}, \mathbf{x}_{j}, \theta\right)\right] .
$$

To provide further insight, Fig. 2 shows the value of $\delta_{\min }\left(\mathbf{x}_{i}, \mathbf{x}_{j}, \theta\right)$ in (7) for BPSK, QPSK, 16-QAM and 64QAM with different $\theta$. If $\theta \in[0, \pi / 2]$, as seen from Fig. $2, \theta$ can be optimized by maximizing the value of $\delta_{\min }\left(\mathbf{x}_{i}, \mathbf{x}_{j}, \theta\right)$ in $(7)$ as

$$
\theta=\left\{\begin{array}{c}
\frac{\pi}{2}, \text { BPSK } \\
\frac{\pi}{4}, \text { QPSK } \\
\frac{\pi}{7} \text { or }\left(\frac{\pi}{2}-\frac{\pi}{7}\right), 16 \mathrm{QAM} \\
\frac{\pi}{16} \text { or }\left(\frac{\pi}{2}-\frac{\pi}{16}\right), 64 \mathrm{QAM}
\end{array} .\right.
$$

\section{Optimal Detector}

ML detector: Let $\mathbf{H} \in \mathbb{C}^{N_{r} \times N_{t}}$ and $\mathbf{n} \in \mathbb{C}^{N_{r} \times 1}$ be the MIMO channel matrix and noise matrix, whose entries are complex-valued Gaussian distributed, yielding $\mathcal{C N}(0,1)$ and $\mathcal{C N}\left(0, \sigma^{2}\right)$, respectively. The received signal $\mathbf{y} \in \mathbb{C}^{N_{r} \times 1}$ can be formulated as

$$
\mathbf{y}=\mathbf{H} \mathbf{x}_{p}+\mathbf{n} .
$$

It follows from Eq. (9) that, the optimal ML-based demodulator can be formulated as

$$
\hat{\mathbf{x}}_{p}=\underset{\mathbf{x}_{p} \in \mathbb{X}_{\text {SM-ATA }}}{\arg \min }\left\|\mathbf{y}-\mathbf{H x}_{p}\right\|_{F}^{2},
$$

where $\mathbb{X}_{\text {SM-ATA }}$ is the set of SM-ATA symbols.

Low-complexity ML detector: Based on the lowcomplexity optimal detector [4] for conventional SM, the low-complexity optimal detector for the proposed SM-ATA scheme can be introduced based on two scenarios. If the value of $N_{t}$ is a power of two, the detector in [4] can be employed for signal detection directly. Otherwise the receiver signal of Eq. (10) is represented as

$$
\mathbf{y}=\left\{\begin{array}{l}
\mathbf{h}_{q} s+\mathbf{n}, \quad \text { if } l \leq N_{t} \\
\mathbf{h}_{q_{l-N_{t}}} s e^{j \theta}+\mathbf{n}, \text { if } l>N_{t}
\end{array} .\right.
$$

For each mapping index $l \in(1, L)$, we can get one symbols as $s_{l}=\mathbb{D}\left(\hat{y}_{l}\right)$, where $\mathbb{D}(\cdot)$ is defined as the digital demodulation function and $\hat{y}_{l}$ is defined as

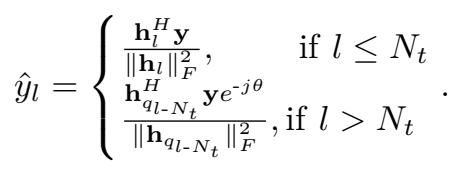

Since we have

$$
\begin{aligned}
& \left\|\mathbf{y}-\mathbf{h}_{q_{l-N}} s_{l} e^{j \theta}\right\|_{F}^{2} \\
& =\|\mathbf{y}\|_{F}^{2}+\left\|\mathbf{h}_{q_{l-N_{t}}} s_{l} e^{j \theta}\right\|_{F}^{2}-2 \Re\left(e^{-j \theta} s_{l}^{H} \mathbf{h}_{q_{l-N_{t}}} \mathbf{y}\right) \\
& =\|\mathbf{y}\|_{F}^{2}+\left\|\mathbf{h}_{q_{l-N_{t}}}\right\|_{F}^{2}\left|s_{l}\right|^{2}-2 \Re\left(s_{l}^{H} \hat{y}_{l}\left\|\mathbf{h}_{q_{l-N_{t}}}\right\|_{F}^{2}\right), \\
& =\|\mathbf{y}\|_{F}^{2}+\left\|\mathbf{h}_{q_{l-N_{t}}}\right\|_{F}^{2}\left(\left|s_{l}\right|^{2}-2 \Re\left(s_{l}^{H} \hat{y}_{l}\right)\right) \\
& =\|\mathbf{y}\|_{F}^{2}+\left\|\mathbf{h}_{q_{l-N_{t}}}\right\|_{F}^{2}\left(\left|\hat{y}_{l}-s_{l}\right|^{2}-\left|\hat{y}_{l}\right|^{2}\right)
\end{aligned}
$$

according to (13), the distance metric $d_{l}$ is defined as

$$
d_{l}=\left\{\begin{array}{ll}
\left\|\mathbf{h}_{l}\right\|_{F}^{2}\left(\left|\hat{y}_{l}-s_{l}\right|^{2}-\left|\hat{y}_{l}\right|^{2}\right), & \text { if } l \leq N_{t} \\
\left\|\mathbf{h}_{q_{l-N_{t}}}\right\|_{F}^{2}\left(\left|\hat{y}_{l}-s_{l}\right|^{2}-\left|\hat{y}_{l}\right|^{2}\right), & \text { if } l>N_{t}
\end{array} .\right.
$$

Then, the optimal mapping index can be estimated as

$$
\hat{l}=\underset{l \in(1, L)}{\arg \min } d_{l} .
$$

Finally, based on the estimated $\hat{l}$ and $s_{\hat{l}}$, the information bits can be recovered based on the SM-ATA mapping rules. Specifically, $s_{\hat{\imath}}$ can be demodulated into bits by APM mapping principle and $\hat{l}$ is demapped into the bits by the extended index group. Taking the extended index group $\Lambda_{p}=\left\{1,2,3,4,5,1 e^{j \theta}, 2 e^{j \theta}, 3 e^{j \theta}\right\}$ for example, the demodulated process for $\hat{l}$ can be expressed as

$$
\begin{aligned}
& \hat{l}=1 \rightarrow 000, \hat{l}=2 \rightarrow 001, \hat{l}=3 \rightarrow 010, \hat{l}=4 \rightarrow 011, \\
& \hat{l}=5 \rightarrow 100, \hat{l}=6 \rightarrow 101, \hat{l}=7 \rightarrow 110, \hat{l}=8 \rightarrow 111 .
\end{aligned}
$$

\section{Complexity Analysis of the Single-RF SM-ATA Detec-} tors

In this section, the complexity of both the fulldimensional ML and of the single-stream ML SM-ATA detectors is analyzed in terms of the numbers of real-valued multiplications and additions. For the specific matrices $\mathbf{A} \in \mathbb{C}^{m \times n}, \mathbf{B} \in \mathbb{C}^{n \times p}, \mathbf{c} \in \mathbb{C}^{n \times 1}$ and $\mathbf{d} \in \mathbb{C}^{n \times 1}$, the operations of $\mathbf{A B},\|\mathbf{c}\|_{F}^{2}$ and $\mathbf{c} \pm \mathbf{d}$ require $(8 m n p-2 m p)$, $(4 n-1)$, and $2 n$ Floating-point operations (Flops), respectively. Accordingly, the complexity order of the ML detector becomes

$$
C_{\mathrm{ML}}^{\mathrm{SM}-\mathrm{ATA}}=\left(12 N_{r}-1\right) \cdot L M,
$$




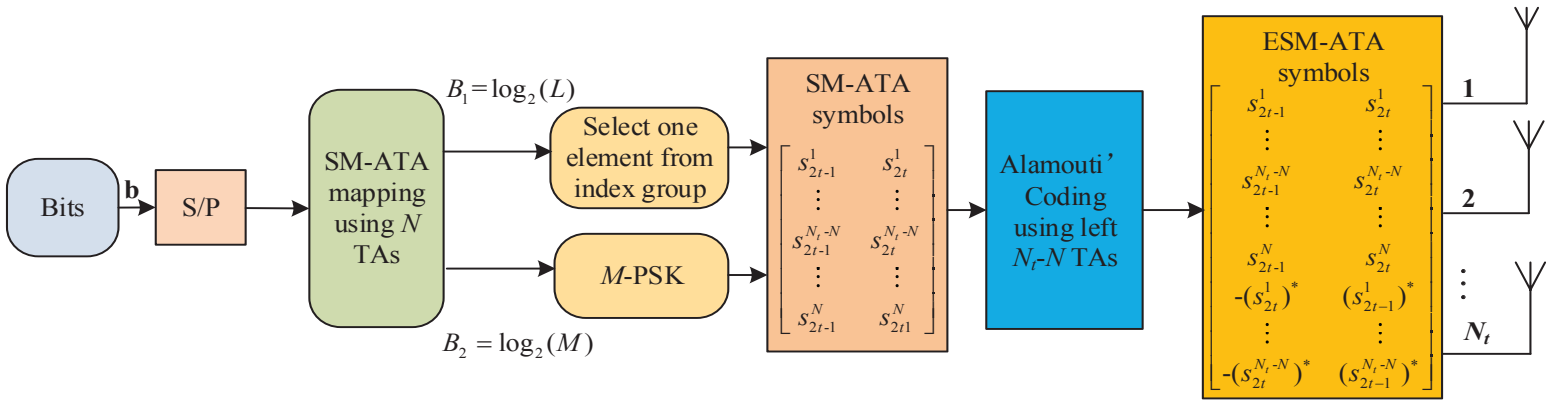

Fig. 3. System model of the proposed ESM-ATA scheme.

TABLE II

ESM-ATA SYMBOL SET FOR THE CASE OF $N_{t}=5, N=3$.

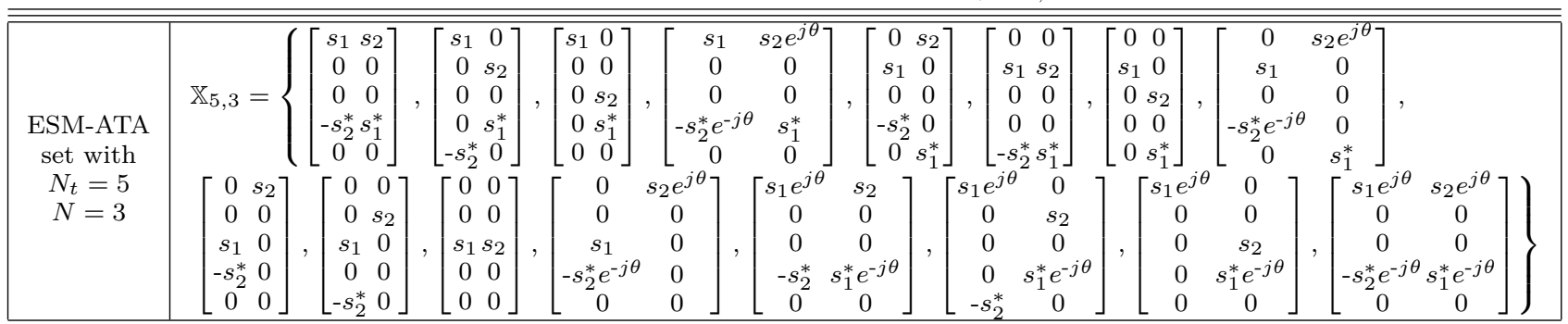

since $\left\|\mathbf{y}-\mathbf{H} \mathbf{x}_{p}\right\|_{F}^{2}$ requires $\left(12 N_{r}-1\right)$ Flops and this operation is computed $L M$ times. And the complexity of the single-stream ML detector is [4]

$$
C_{\mathrm{SS}-\mathrm{ML}}^{\mathrm{SM}}=11 N_{r} L
$$

IV. Twin-RF Enhanced Spatial Modulation for An Arbitrary Number of Transmit Antennas

To improve the diversity gain of the SM-ATA scheme, the ESM-ATA is proposed for an arbitrary number of TAs by invoking the space-time block coding principle. For any number of TAs, the $N_{t}$ TAs are divided into two sets: the first $N\left(N<N_{t}\right)$ TAs are used for conveying a SMATA symbol, while the remaining $\left(N_{t}-N\right)$ TAs are used for enhancing the SM-ATA symbol's integrity by invoking space-time block coding. Explicitly, Table II presents the ESM-ATA symbol sets for the cases of $N_{t}=5, N=3$, where the extended index group of the SM-ATA scheme associated with $N=3$ is $\Lambda_{p}=\left\{1,2,3,1 e^{j \theta}\right\}$, while $s_{1}$ and $s_{2}$ denote the $M$-ary APM symbols. As seen from Table II, the ESM-ATA symbol sets are obtained based on the SMATA symbol set consisting of two time slots. For the case of $N_{t}=5, N=3$, the fourth TA and fifth TAs are utilized for invoking Alamouti's space-time block code in conjunction with the first and second TAs. Next, the generalized ESMATA system model and our low-complexity detector are detailed as follows.

\section{A. Generalized ESM-ATA Transceiver Design}

We consider an ESM-ATA system associated with $N_{t}$ TAs and $N_{r}$ receiver antennas communicating over flat Rayleigh fading channels. The system model of the proposed ESM-ATA scheme is shown in Fig. 3, which operates as follows.

Step 1: Determine the index group.
1) If $\log _{2}(N)$ is an integer, then the index group is $\Lambda_{p}=$ $\{1,2, \ldots, N\}$.

2) Otherwise, the size of the new index group is extended to $L=2^{\left\lceil\log _{2}\left(N_{t}\right)\right\rceil}$, which is formulated as $\Lambda_{p}=\left\{1, \ldots, N, 1 e^{j \theta}, \ldots,(L-N) e^{j \theta}\right\}$ for simplicity.

Step 2: SM-ATA mapping using $N$ TAs and formulating the SM-ATA symbols at the $(2 t-1)$-st and $2 t$-th time slots as

$$
\mathbf{X}_{N}=\left[\mathbf{x}_{N}^{2 t-1}, \mathbf{x}_{N}^{2 t}\right]_{N \times 2}
$$

with

$$
\begin{aligned}
& \mathbf{x}_{N}^{2 t-1}=\left[s_{2 t-1}^{1}, \ldots, s_{2 t-1}^{N_{t-N}}, \ldots, s_{2 t-1}^{N}\right]^{T}=[\underbrace{0, \ldots, 0}_{q_{2 t-1}-1}, s_{2 t-1}^{q_{2 t-1}} \underbrace{0, \ldots, 0}_{N-q_{2 t-1}}]^{T}, \\
& \mathbf{x}_{N}^{2 t}=\left[s_{2 t}^{1}, \ldots, s_{2 t}^{N_{t}-N}, \ldots, s_{2 t}^{N}\right]^{T}=[\underbrace{0, \ldots, 0}_{q_{2 t}-1}, s_{2 t}^{q_{2 t}} \underbrace{0, \ldots, 0}_{N-q_{2 t}}]^{T},
\end{aligned}
$$

with $s_{2 t-1}^{q_{2 t-1}}, s_{2 t}^{q_{2 t}} \in\left\{\mathcal{S}, \mathcal{S} e^{j \theta}\right\}$ and $q_{2 t-1}=(1, \ldots, N)$ and $q_{2 t}=(1, \ldots, N)$ representing the activated TA indices of the SM-ATA symbols $\mathbf{x}_{N}^{2 t-1}$ and $\mathbf{x}_{N}^{2 t}$, respectively. $\mathcal{S}$ is the set of constellation symbols.

Step 3: Encoded the first $\left(N_{t}-N\right)$ symbols of $\mathbf{x}_{N}^{2 t-1}$ and $\mathbf{x}_{N}^{2 t-1}$ with Almouti's STBC and transmitted by the left $\left(N_{t}-N\right)$ TAs.

Step 4: Formulate the ESM-ATA symbols as

$$
\mathbf{X}_{N_{t}}=\left[\mathbf{x}_{N_{t}}^{2 t-1}, \mathbf{x}_{N_{t}}^{2 t}\right]_{N_{t} \times 2}
$$

with

$$
\begin{aligned}
& \mathbf{x}_{N_{t}}^{2 t-1}=\left[s_{2 t-1}^{1}, \ldots, s_{2 t-1}^{N_{t}-N}, \ldots, s_{2 t-1}^{N},-\left(s_{2 t}^{1}\right)^{*} \ldots,-\left(s_{2 t}^{N_{t}-N}\right)^{*}\right]^{T} \\
& \mathbf{x}_{N_{t}}^{2 t}=\left[s_{2 t}^{1}, \ldots, s_{2 t}^{N_{t}-N}, \ldots, s_{2 t}^{N},\left(s_{2 t-1}^{1}\right)^{*} \ldots,\left(s_{2 t-1}^{N_{t}-N}\right)^{*}\right]^{T} .
\end{aligned}
$$


According to (21), the terms $\mathbf{x}_{N_{t}}^{2 t-1}$ and $\mathbf{x}_{N_{t}}^{2 t}$ can be further expressed as

$$
\begin{aligned}
& \mathbf{x}_{N_{t}}^{2 t-1}= \begin{cases}{\left[\ldots, 0, s_{2 t-1}^{q_{2 t-1}}, \ldots, 0,-\left(s_{2 t}^{q_{2 t}}\right)^{*}, \ldots, 0\right]^{T},} & \text { if } q_{2 t} \leq N_{t^{-}}-N \\
{\left[0, \ldots, 0, s_{2 t-1}^{q_{2 t-1}}, 0, \ldots, 0\right]^{T},} & \text { if } q_{2 t}>N_{t^{-}}-N\end{cases} \\
& \mathbf{x}_{N_{t}}^{2 t}= \begin{cases}{\left[\ldots, 0, s_{2 t}^{2 t}, \ldots, 0,\left(s_{2 t-1}^{q_{2 t-1}}\right)^{*}, \ldots, 0\right]^{T},} & \text { if } q_{2 t-1} \leq N_{t^{-}}-N \\
{\left[0, \ldots, 0, s_{2 t}^{q_{2 t}}, 0, \ldots, 0\right]^{T},} & \text { if } q_{2 t-1}>N_{t^{-}}\end{cases}
\end{aligned}
$$

Accordingly, there are at most two nonzero elements for the ESM-ATA symbols $\mathbf{x}_{N_{t}}^{2 t-1}$ and $\mathbf{x}_{N_{t}}^{2 t}$.

\section{B. Diversity and Coding gain of the twin-RF ESM-ATA}

In the proposed ESM-ATA scheme, the left $\left(N_{t}-N\right)$ TAs are used for Alamouti's STBC coding, which is capable of improving the diversity gain of the SM-ATA scheme. The diversity order and the coding gain are dominated by the value of $N$. Assuming that $\mathbf{X}_{i}=\left[\mathbf{x}_{1, i}, \mathbf{x}_{2, i}\right] \in \mathbb{X}_{\mathrm{ESM}}$ and $\mathbf{X}_{j}=\left[\mathbf{x}_{1, j}, \mathbf{x}_{2, j}\right] \in \mathbb{X}_{\mathrm{ESM}}$ are the ESM-ATA symbols, the coding gain of the ESM-ATA scheme is defined by

$$
G_{E}=\min _{\mathbf{X}_{i} \neq \mathbf{X}_{j}}\left|\operatorname{det} \Delta_{E}^{H} \Delta_{E}\right|,
$$

where we have $\Delta_{E}=\left(\mathbf{X}_{i}-\mathbf{X}_{j}\right)$. The diversity order is the rank of $\Delta_{E}^{H} \Delta_{E}$, which is different from the value of $N$.

Case $1\left(N_{t}-N\right)<N$ : For the case of $\left(N_{t}-N\right)<N$, the subsets of the ESM-ATA set $\mathbb{X}_{\mathrm{ESM}}=\left\{\mathbb{X}_{1}, \mathbb{X}_{2}, \mathbb{X}_{3}, \mathbb{X}_{4}\right\}$ can be expressed as

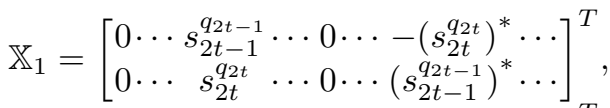

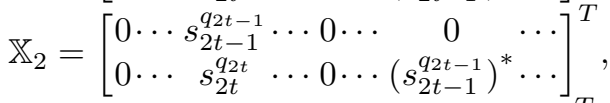

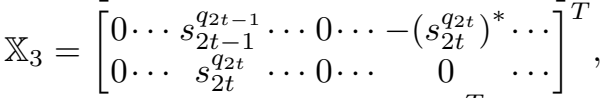

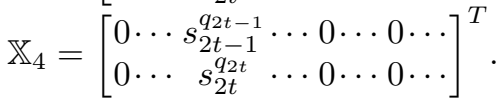

Hence, the coding gain of the ESM-ATA scheme can be expressed as

$$
G_{E}=\min _{\mathbf{X}_{i} \neq \mathbf{X}_{j}}\left|\operatorname{det} \Delta_{E}^{H} \Delta_{E}\right|=\min _{\mathbf{X}_{i} \neq \mathbf{X}_{j}, \mathbf{X}_{i}, \mathbf{X}_{j} \in \mathbb{X}_{4}}\left|\operatorname{det} \Delta_{E}^{H} \Delta_{E}\right|=0,
$$

where the rank of $\Delta_{E}^{H} \Delta_{E}$ in this case is one, hence the diversity order of ESM-ATA becomes one for the case of $\left(N_{t}-N\right)<N$. Additionally, in this case, for each time slot, the probability of the ESM-ATA symbol having two nonzero elements is equal to $P_{2}=\frac{N_{t}-N+L-\left\lfloor\log _{2}(N)\right\rfloor}{L}$ and that of having one nonzero element is equal to $P_{1}=1-P_{2}$. Assuming that $\left|s_{2 t-1}^{q_{2 t-1}}\right|^{2}=\left|\left(s_{2 t}^{q_{2 t}}\right)\right|^{2}=1$, the average energy consumption $P_{e}$ is equal to $P_{e}=2 P_{2}+P_{1}$, which is satisfied $1<P_{e}<2$.

Case $2\left(N_{t}-N\right) \geq N$ : For the case of $\left(N_{t}-N\right) \geq N$, the ESM-ATA symbols $\mathbf{X}_{i}$ and $\mathbf{X}_{j}$ can be further expressed for the case of $\left(N_{t}-N\right)=N$ as

$$
\mathbf{X}_{i}=\left[\begin{array}{cc}
\mathbf{x}_{N, i}^{1} & \mathbf{x}_{N, i}^{2} \\
\left(\mathbf{x}_{N, i}^{2}\right)^{*} & \left(\mathbf{x}_{N, i}^{1}\right)^{*}
\end{array}\right], \mathbf{X}_{j}=\left[\begin{array}{cc}
\mathbf{x}_{N, j}^{1} & \mathbf{x}_{N, j}^{2} \\
\left(\mathbf{x}_{N, j}^{2}\right)^{*} & \left(\mathbf{x}_{N, j}^{1}\right)^{*}
\end{array}\right],
$$

or for the case of $\left(N_{t}-N\right)>N$ as

$$
\mathbf{X}_{i}=\left[\begin{array}{cc}
\mathbf{x}_{N, i}^{1} & \mathbf{x}_{N, i}^{2} \\
\left(\mathbf{x}_{N, i}^{2}\right)^{*} & \left(\mathbf{x}_{N, i}^{1}\right)^{*} \\
\mathbf{O}_{N_{t}-2 N} & \mathbf{O}_{N_{t}-2 N}
\end{array}\right], \mathbf{X}_{j}=\left[\begin{array}{cc}
\mathbf{x}_{N, j}^{1} & \mathbf{x}_{N, j}^{2} \\
\left(\mathbf{x}_{N, j}^{2}\right)^{*} & \left(\mathbf{x}_{N, j}^{1}\right)^{*} \\
\mathbf{O}_{N_{t}-2 N} & \mathbf{O}_{N_{t}-2 N}
\end{array}\right],
$$

where $\mathbf{x}_{N, i}^{1}$ and $\mathbf{x}_{N, i}^{2}$ denote the first and second SM-ATA symbol in the ESM-ATA symbol $\mathbf{X}_{i}$, while $\mathbf{x}_{N, j}^{1}$ and $\mathbf{x}_{N, j}^{2}$ denote the first and second SM-ATA symbol in the ESMATA symbol $\mathbf{X}_{j}$. The coding gain of the ESM-ATA scheme associated with $\left(N_{t}-N\right)=N$ and $\left(N_{t}-N\right)>N$ can be expressed as

$$
\begin{aligned}
G_{E} & =\min _{\mathbf{X}_{i} \neq \mathbf{X}_{j}}\left|\operatorname{det}\left(\Delta_{E}^{H} \Delta_{E}\right)\right| \\
& =\min _{\mathbf{X}_{i} \neq \mathbf{X}_{j}}\left[\left(\mathbf{x}_{N, i}^{1}-\left.\mathbf{x}_{N, j}^{1}\right|^{2}+\left|\mathbf{x}_{N, i}^{2}-\mathbf{x}_{N, j}^{2}\right|^{2}\right)^{2}\right], \\
& \neq 0
\end{aligned}
$$

and the rank of $\Delta_{E}^{H} \Delta_{E}$ in this case is two, hence the diversity order of ESM-ATA is two for the case of $\left(N_{t}-N\right) \geq$ $N$. Additionally, in this case, assuming that $\left|\mathbf{x}_{N, i}^{1}\right|^{2}=$ $\left|\mathbf{x}_{N, i}^{2}\right|^{2}=1$, the average energy consumption becomes two.

\section{Optimization of the twin-RF ESM-ATA}

In this section, the power allocation of the twin-RF ESM-ATA is further discussed and optimized by maximizing the coding gain. Since the ESM-ATA scheme having an even number of TAs associated with $N=N_{t} / 2$ can achieve the same diversity order and coding gain as the ESM-ATA scheme having an odd number of TAs with $N=\left(N_{t}-1\right) / 2$, we mainly discuss the ESM-ATA schemes having an even number of TAs with $N=N_{t} / 2$ for simplicity. Assuming that the power of the first SM-ATA symbol is $\sqrt{\rho}$ and the second SM-ATA symbol is $\sqrt{2-\rho}$, the ESM-ATA symbols $\mathbf{X}_{i}$ and $\mathbf{X}_{j}$ can be further expressed as

$$
\begin{aligned}
\mathbf{X}_{i} & =\left[\begin{array}{cc}
\sqrt{\rho} \mathbf{x}_{N, i}^{1} & \sqrt{2-\rho} \mathbf{x}_{N, i}^{2} \\
\sqrt{2-\rho}\left(\mathbf{x}_{N, i}^{2}\right)^{*} & \sqrt{\rho}\left(\mathbf{x}_{N, i}^{1}\right)^{*}
\end{array}\right], \\
\mathbf{X}_{j} & =\left[\begin{array}{cc}
\sqrt{\rho} \mathbf{x}_{N, j}^{1} & \sqrt{2-\rho} \mathbf{x}_{N, j}^{2} \\
\sqrt{2-\rho}\left(\mathbf{x}_{N, j}^{2}\right)^{*} & \sqrt{\rho}\left(\mathbf{x}_{N, j}^{1}\right)^{*}
\end{array}\right],
\end{aligned}
$$

while $\Delta_{E}^{H} \Delta_{E}$ can be computed as

$$
\Delta_{E}^{H} \Delta_{E}=\left[\begin{array}{cc}
A_{E} & 0 \\
0 & A_{E}
\end{array}\right]
$$

with $A_{E}=\rho\left|\mathbf{x}_{N, i}^{1}-\mathbf{x}_{N, j}^{1}\right|^{2}+(2-\rho)\left|\mathbf{x}_{N, i}^{2}-\mathbf{x}_{N, j}^{2}\right|^{2}$, which can be further expressed as

$A_{E}= \begin{cases}0, & \text { if } \mathbf{x}_{N, i}^{1}=\mathbf{x}_{N, j}^{1}, \mathbf{x}_{N, i}^{2}=\mathbf{x}_{N, j}^{2} \\ \rho\left|\mathbf{x}_{N, i}^{1}-\mathbf{x}_{N, j}^{1}\right|^{2}, \quad \text { if } \mathbf{x}_{N, i}^{1} \neq \mathbf{x}_{N, j}^{1}, \mathbf{x}_{N, i}^{2}=\mathbf{x}_{N, j}^{2} \\ (2-\rho)\left|\mathbf{x}_{N, i}^{2}-\mathbf{x}_{N, j}^{2}\right|^{2}, & \text { if } \mathbf{x}_{N, i}^{1}=\mathbf{x}_{N, j}^{1}, \mathbf{x}_{N, i}^{2} \neq \mathbf{x}_{N, j}^{2} \\ \rho\left|\mathbf{x}_{N, i}^{1}-\mathbf{x}_{N, j}^{1}\right|^{2}+(2-\rho)\left|\mathbf{x}_{N, i}^{2}-\mathbf{x}_{N, j}^{2}\right|^{2}, \text { Otherwise. }\end{cases}$

Hence, the coding gain can be expressed as

$$
G_{E}=\min _{\mathbf{X}_{i} \neq \mathbf{X}_{j}}\left|\operatorname{det}\left(\Delta_{E}^{H} \Delta_{E}\right)\right|=\min _{\mathbf{X}_{i} \neq \mathbf{X}_{j}}\left(A_{E}^{2}\right) .
$$

According to (33), we have

$$
\begin{aligned}
& \min _{\mathbf{x}_{i} \neq \mathbf{X}_{j}}\left(A_{E}\right)=\min \left[\min _{\mathbf{x}_{N, i}^{1} \neq \mathbf{x}_{N, j}^{1}}\left(\rho\left|\mathbf{x}_{N, i}^{1}-\mathbf{x}_{N, j}^{1}\right|^{2}\right),\right. \\
& \left.\min _{\mathbf{x}_{N, i}^{1} \neq \mathbf{x}_{N, j}^{1}}\left((2-\rho)\left|\mathbf{x}_{N, i}^{2}-\mathbf{x}_{N, j}^{2}\right|^{2}\right)\right]
\end{aligned}
$$


TABLE III

MAXIMUM CODING GAIN COMPARISONS BETWEEN THE PROPOSED ESM-ATA SCHEMES AND CONVENTIONAL STBC-SM SCHEMES

\begin{tabular}{|c|c|c|c|}
\hline \multicolumn{4}{|c|}{$N_{t}=4, N=2$} \\
\hline & Rate $=3$ & Rate $=4$ & Rate $=5$ \\
\hline SM-STBC [30] & 2.6863 & 0.3431 & 0.0808 \\
\hline M-SM-STBC [33] & 2.9922 & 0.3431 & 0.1146 \\
\hline Proposed ESM-ATA & 4 & 0.3431 & 0.16 \\
\hline \multicolumn{4}{|c|}{$N_{t}=6, N=3$} \\
\hline & Rate $=4$ & Rate $=5$ & Rate $=6$ \\
\hline SM-STBC [30] & 0.3431 & 0.0287 & $2.418 \times 10^{-4}$ \\
\hline M-SM-STBC [33] & 0.3431 & 0.0649 & 0.0011 \\
\hline Proposed ESM-ATA & 0.3431 & 0.0232 & 0.0014 \\
\hline \multicolumn{4}{|c|}{$N_{t}=8, N=4$} \\
\hline & Rate $=4$ & Rate $=5$ & Rate $=6$ \\
\hline SM-STBC [30] & 1.2179 & 0.3074 & 0.0487 \\
\hline M-SM-STBC [33] & 1.2773 & 0.3074 & 0.0487 \\
\hline Proposed ESM-ATA & 4 & 0.3431 & 0.16 \\
\hline
\end{tabular}

Since we have

$$
\min _{\mathbf{x}_{N, i}^{1} \neq \mathbf{x}_{N, j}^{1}}\left(\left|\mathbf{x}_{N, i}^{1}-\mathbf{x}_{N, j}^{1}\right|^{2}\right)=\min _{\mathbf{x}_{N, i}^{2} \neq \mathbf{x}_{N, j}^{2}}\left(\left|\mathbf{x}_{N, i}^{2}-\mathbf{x}_{N, j}^{2}\right|^{2}\right),
$$

the coding gain can be further expressed as

$$
G_{E}=\min _{\mathbf{X}_{i} \neq \mathbf{X}_{j}}\left[\rho^{2} \delta_{\min }^{2},(2-\rho)^{2} \delta_{\min }^{2}\right],
$$

where $\delta_{\min }=\min _{\mathbf{x}_{N, i}^{1} \neq \mathbf{x}_{N, j}^{1}}\left(\left|\mathbf{x}_{N, i}^{1}-\mathbf{x}_{N, j}^{1}\right|^{2}\right)$ and can be obtained by (6) and (7) as

$$
\delta_{\min }=\left\{\begin{array}{l}
\min _{s_{i}^{1} \neq s_{j}^{1}}\left(\left|s_{i}^{1}-s_{j}^{1}\right|^{2}\right), \text { if } N \text { is a power of } 2, \\
\min _{\forall s_{i}^{1}, s_{j}^{1}}\left(\left|s_{i}^{1}-s_{j}^{1} e^{j \theta}\right|^{2}\right), \text { else. }
\end{array}\right.
$$

where $s_{i}^{1}, s_{j}^{1}$ are the constellation symbols of $\mathbf{x}_{N, i}^{1}, \mathbf{x}_{N, j}^{1}$. As a result, $\rho$ is equal to one to obtain the maximum coding gain $G_{E}$ as

$$
G_{E}^{\max }=\left\{\begin{array}{l}
\min _{s_{i}^{1} \neq s_{j}^{1}}\left(\left|s_{i}^{1}-s_{j}^{1}\right|^{4}\right), \text { if } N \text { is a power of } 2, \\
\min _{\forall s_{i}^{1}, s_{j}^{1}}\left(\left|s_{i}^{1}-s_{j}^{1} e^{j \theta}\right|^{4}\right), \text { else. }
\end{array}\right.
$$

where $\theta$ can be obtained via (9).

In short, for a given constellation point set, the coding gain of the proposed twin-RF ESM-ATA schemes remains constant as $N_{t}$ increases. Table III compares the coding gain of ESM-ATA schemes to that of the conventional STBC-SM and MC-STBC-SM schemes. As seen from Table III, for the case of $N$ being a power of two, the coding gain of the proposed ESM-ATA is significantly higher than that of the classic STBC-SM schemes and the coding gain gap increases, as $N_{t}$ increases. When $N$ is not an integer power of two, the proposed ESM-ATA scheme offers a similar coding gain to that of the conventional STBC-SM schemes.

\section{Optimal and Suboptimal Detectors}

Optimal ML-based Detector: In this section, the ML detector of the proposed ESM-ATA scheme is introduced. According to (10), the received signal matrix $\mathbf{Y}_{t} \in \mathbb{C}^{N_{r} \times 2}$ consisting of two time slots can be expressed as

$$
\mathbf{Y}_{t}=\left[\mathbf{y}_{2 t-1}, \mathbf{y}_{2 t}\right]=\mathbf{H}\left[\mathbf{x}_{N_{t}}^{2 t-1}, \mathbf{x}_{N_{t}}^{2 t}\right]+\left[\mathbf{n}_{2 t-1}, \mathbf{n}_{2 t}\right] .
$$

Hence, the optimal ML-based demodulator can be formulated as

$$
\hat{\mathbf{X}}_{N_{t}}=\left[\hat{\mathbf{x}}_{N_{t}}^{2 t-1}, \hat{\mathbf{x}}_{N_{t}}^{2 t}\right]=\underset{\mathbf{X}_{N_{t}} \in \mathbb{X}_{\mathrm{ESM}}}{\arg \min }\left\|\mathbf{Y}_{t}-\mathbf{H} \mathbf{X}_{N_{t}}\right\|_{F}^{2},
$$

where $\mathbb{X}_{\mathrm{ESM}}$ is the set of the proposed ESM-ATA symbols. Observe that the complexity of the ML detector significantly increases with the parameters $N_{t}$ and $M$.

Low-complexity Suboptimal Detector: In this section, a low-complexity suboptimal detector is proposed for the ESM-ATA system. For the proposed ESM-ATA system associated with $\left(N_{t}, N_{r}, N, M\right)$, both the transmission rate and the performance attained are dominated by the value of $N$, hence the low-complexity suboptimal detector is designed based on two different scenarios.

Case 1: $\log _{2}(N)$ is an integer. In this case, the transmission rate of ESM-ATA is $R_{\mathrm{ESM}}=\log _{2}(N)+\log _{2}(M)$. The size of the index group is $L=2^{\log _{2}(N)}$, which is expressed as $\Lambda_{p}=\{1,2, \ldots, N\}$. In this case, the mapping index $l$ is always the same as the activated TA index $q$ and the $M$-ary APM symbols do not have to be rotated by $\theta$. According to (40), the received signal $\mathbf{y}_{2 t-1}$ and $\mathbf{y}_{2 t}$ in $\mathbf{Y}_{t} \in \mathbb{C}^{N_{r} \times 2}$ can be represented as

$$
\begin{aligned}
& \mathbf{y}_{2 t-1}= \begin{cases}\mathbf{h}_{q_{2 t-1}} s_{2 t-1}^{q_{2 t-1}}-\mathbf{h}_{q_{2 t}+N}\left(s_{2 t}^{q_{2 t}}\right)^{*}+\mathbf{n}_{2 t-1}, & \text { if } q_{2 t} \leq N_{t^{-}} N \\
\mathbf{h}_{q_{2 t-1}} s_{2 t-1}^{q_{2 t-1}}+\mathbf{n}_{2 t-1}, & \text { if } q_{2 t}>N_{t^{-}} N\end{cases} \\
& \mathbf{y}_{2 t}= \begin{cases}\mathbf{h}_{q_{2 t}} s_{2 t}^{q_{2 t}}+\mathbf{h}_{q_{2 t-1}+N}\left(s_{2 t-1}^{q_{2 t-1}}\right)^{*}+\mathbf{n}_{2 t}, & \text { if } q_{2 t-1} \leq N_{t^{-}} N \\
\mathbf{h}_{q_{2 t}} s_{2 t}^{q_{22}}+\mathbf{n}_{2 t}, & \text { if } q_{2 t-1}>N_{t^{-}} N\end{cases}
\end{aligned}
$$

Hence, the signal matrix $\left[\mathbf{y}_{2 t-1}, \mathbf{y}_{2 t}^{*}\right]$ can be expressed as

$$
\left[\begin{array}{l}
\mathbf{y}_{2 t-1} \\
\mathbf{y}_{2 t}^{*}
\end{array}\right]=\mathbf{W}_{t}\left[\begin{array}{l}
s_{2 t-1}^{q_{2 t-1}} \\
\left(s_{2 t}^{q_{2 t}}\right)^{*}
\end{array}\right]+\left[\begin{array}{l}
\mathbf{n}_{2 t-1} \\
\mathbf{n}_{2 t}^{*}
\end{array}\right]
$$

where we have

$$
\mathbf{W}_{t}=\left\{\begin{array}{l}
{\left[\begin{array}{cc}
\mathbf{h}_{q_{2 t-1}} & -\mathbf{h}_{q_{2 t}+N} \\
\mathbf{h}_{q_{2 t-1}+N}^{*} & \mathbf{h}_{q_{2 t}}^{*}
\end{array}\right], \text { if } q_{2 t}, q_{2 t-1} \leq N_{t^{-}} N} \\
{\left[\begin{array}{cc}
\mathbf{h}_{q_{2 t-1}} & \mathbf{O} \\
\mathbf{h}_{q_{2 t-1}+N}^{*} \mathbf{h}_{q_{2 t}}^{*}
\end{array}\right], \text { if } q_{2 t-1} \leq N_{t^{-}} N, q_{2 t}>N_{t^{-}}} \\
{\left[\begin{array}{cc}
\mathbf{h}_{q_{2 t-1}}-\mathbf{h}_{q_{2 t}+N} \\
\mathbf{O} & \mathbf{h}_{q_{2 t}}^{*}
\end{array}\right], \text { if } q_{2 t-1}>N_{t^{-}} N, q_{2 t} \leq N_{t^{-}}} \\
{\left[\begin{array}{cc}
\mathbf{h}_{q_{2 t-1}} & \mathbf{O} \\
\mathbf{O} & \mathbf{h}_{q_{2 t}}^{*}
\end{array}\right], \quad \text { if } q_{2 t-1}>N_{t^{-}} N, q_{2 t}>N_{t^{-}} N}
\end{array} .\right.
$$

Then signal detection can be performed based on Eq. (43). Since we have $q_{2 t-1}, q_{2 t} \in \Lambda_{p}=\{1,2, \ldots, N\}$, there are a total number of $N^{2}$ index combinations $\mathbb{I}$ formulated as

$$
\mathbb{I}=\{\underbrace{(1,1)}_{I_{1}}, \underbrace{(1,2)}_{I_{2}}, \ldots, \underbrace{(1, N)}_{I_{N}}, \ldots, \underbrace{(N, N)}_{I_{N}}\} .
$$

For each index combination $\left(q_{2 t-1}, q_{2 t}\right) \in \mathbb{I}_{m} m \in\left(1, N^{2}\right)$, we can obtain the corresponding channel matrix $\mathbf{W}_{t}^{m}$ according to (44). Then the associated symbol vector can be estimated as

$$
\hat{\mathbf{s}}_{t}^{m}=\left[\begin{array}{l}
\hat{s}_{2 t-1}^{q_{2 t-1}} \\
\left(\hat{s}_{2 t}^{q_{2 t}}\right)^{*}
\end{array}\right]=\mathbb{D}\left(\left(\mathbf{W}_{t}^{m}\right)^{\dagger}\left[\begin{array}{l}
\mathbf{y}_{2 t-1} \\
\mathbf{y}_{2 t}^{*}
\end{array}\right]\right),
$$

where $\left(\mathbf{W}_{t}^{m}\right)^{\dagger}=\left(\left(\mathbf{W}_{t}^{m}\right)^{H} \mathbf{W}_{t}^{m}+\sigma^{2} \mathbf{I}_{2}\right)^{-1}\left(\mathbf{W}_{t}^{m}\right)^{H}$. After obtaining $N^{2}$ symbol vectors as $\left(I_{1}, \hat{\mathbf{s}}_{t}^{1}\right), \ldots,\left(I_{N^{2}}, \hat{\mathbf{s}}_{t}^{N^{2}}\right)$, we opt 


$$
\begin{gathered}
\mathbf{y}_{2 t-1}= \begin{cases}\mathbf{h}_{q_{2 t-1}} s_{2 t-1}-\mathbf{h}_{q_{2 t}+N} s_{2 t}^{*}+\mathbf{n}_{2 t-1}, & \text { if } q_{2 t} \leq N_{t}-N, l_{2 t} \leq N, l_{2 t-1} \leq N \\
\mathbf{h}_{q_{2 t-1}} s_{2 t-1}-\mathbf{h}_{q_{2 t}+N}\left(s_{2 t} e^{j \theta}\right)^{*}+\mathbf{n}_{2 t-1}, & \text { if } q_{2 t} \leq N_{t}-N, l_{2 t}>N, l_{2 t-1} \leq N \\
\mathbf{h}_{q_{2 t-1}} s_{2 t-1} e^{j \theta}-\mathbf{h}_{q_{2 t}+N} s_{2 t}^{*}+\mathbf{n}_{2 t-1}, & \text { if } q_{2 t} \leq N_{t}-N, l_{2 t} \leq N, l_{2 t-1}>N \\
\mathbf{h}_{q_{2 t-1}} s_{2 t-1} e^{j \theta}-\mathbf{h}_{q_{2 t}+N}\left(s_{2 t} e^{j \theta}\right)^{*}+\mathbf{n}_{2 t-1}, & \text { if } q_{2 t} \leq N_{t}-N, l_{2 t}>N, l_{2 t-1}>N \\
\mathbf{h}_{q_{2 t-1}} s_{2 t-1}+\mathbf{n}_{2 t-1}, & \text { if } q_{2 t}>N_{t}-N, l_{2 t-1} \leq N, \\
\mathbf{h}_{q_{2 t-1}} s_{2 t-1} e^{j \theta}+\mathbf{n}_{2 t-1}, & \text { if } q_{2 t}>N_{t}-N, l_{2 t-1}>N\end{cases} \\
\mathbf{y}_{2 t}= \begin{cases}\mathbf{h}_{q_{2 t}} s_{2 t}+\mathbf{h}_{q_{2 t-1}+N} s_{2 t-1}^{*}+\mathbf{n}_{2 t}, & \text { if } q_{2 t-1} \leq N_{t}-N, l_{2 t-1} \leq N, l_{2 t} \leq N \\
\mathbf{h}_{q_{2 t}} s_{2 t}+\mathbf{h}_{q_{2 t-1}+N}\left(s_{2 t-1} e^{j \theta}\right)^{*}+\mathbf{n}_{2 t}, & \text { if } q_{2 t-1} \leq N_{t}-N, l_{2 t-1}>N, l_{2 t} \leq N \\
\mathbf{h}_{q_{2 t}} s_{2 t} e^{j \theta}+\mathbf{h}_{q_{2 t-1}+N} s_{2 t-1}^{*}+\mathbf{n}_{2 t}, & \text { if } q_{2 t-1} \leq N_{t}-N, l_{2 t-1} \leq N, l_{2 t}>N \\
\mathbf{h}_{q_{2 t}} s_{2 t} e^{j \theta}+\mathbf{h}_{q_{2 t-1}+N}\left(s_{2 t-1} e^{j \theta}\right)^{*}+\mathbf{n}_{2 t}, & \text { if } q_{2 t-1} \leq N_{t}-N, l_{2 t-1}>N, l_{2 t}>N \\
\mathbf{h}_{q_{2 t}} s_{2 t}+\mathbf{n}_{2 t}, & \text { if } q_{2 t-1}>N_{t}-N, l_{2 t} \leq N \\
\mathbf{h}_{q_{2 t}} s_{2 t} e^{j \theta}+\mathbf{n}_{2 t}, & \text { if } q_{2 t-1}>N N^{-N}, l_{2 t}>N .\end{cases}
\end{gathered}
$$

for the best one from the $N^{2}$ symbol vectors according to:

$$
\hat{m}=\arg \min _{m \in\left(1, N^{2}\right)}\left\|\left[\begin{array}{l}
\mathbf{y}_{2 t-1} \\
\mathbf{y}_{2 t}^{*}
\end{array}\right]-\mathbf{W}_{t}^{m} \hat{\mathbf{s}}_{t}^{m}\right\|_{F}^{2} .
$$

Consequently, the final estimated signal can be expressed as $\left(I_{\hat{m}}, \hat{\mathbf{s}}_{t}^{\hat{m}}\right)$, which can then be demodulated to generate the information bits by the SM-ATA mapping rule.

Case 2: $\log _{2}(N)$ is not an integer. In this case, the transmission rate of ESM-ATA is $R_{\mathrm{ESM}}=$ $\left\lfloor\log _{2}(N)\right\rfloor+1+\log _{2}(M)$. The index group is expressed as $\Lambda_{p}=\left\{1,2, \ldots, N, 1 e^{j \theta}, 2 e^{j \theta}, \ldots,(L-N) e^{j \theta}\right\}$. Assuming that $l_{2 t-1}$ and $l_{2 t}$ are the mapping indices of the SM-ATA symbols $\mathbf{x}_{N}^{2 t-1}$ and $\mathbf{x}_{N}^{2 t}$ in Eq. (20), respectively and $s_{2 t-1}$ and $s_{2 t}$ are the modulated $M$-ary APM symbols of $\mathbf{x}_{N}^{2 t-1}$ and $\mathbf{x}_{N}^{2 t}$, the relationships between $l_{2 t-1}, l_{2 t}$ and $q_{2 t-1}, q_{2 t}$ can be expressed as

$$
l_{2 t-1}=\left\{\begin{array}{ll}
q_{2 t-1}, & \text { if } l_{2 t-1} \leq N \\
q_{2 t-1}+N, & \text { if } l_{2 t-1}>N
\end{array}, l_{2 t}=\left\{\begin{array}{l}
q_{2 t}, \quad \text { if } l_{2 t} \leq N \\
q_{2 t}+N, \text { if } l_{2 t}>N
\end{array},\right.\right.
$$

while the relationships between $s_{2 t-1}^{q_{2 t-1}}, s_{2 t}^{q_{2 t}}$ and $s_{2 t-1}, s_{2 t}$ are formulated as:

$$
\begin{aligned}
& s_{2 t-1}^{q_{2 t-1}}= \begin{cases}s_{2 t-1}, & \text { if } l_{2 t-1} \leq N \\
s_{2 t-1} e^{j \theta}, & \text { if } l_{2 t-1}>N\end{cases} \\
& s_{2 t}^{q_{2 t}}=\left\{\begin{array}{ll}
s_{2 t}, & \text { if } l_{2 t} \leq N \\
s_{2 t} e^{j \theta}, & \text { if } l_{2 t}>N
\end{array} .\right.
\end{aligned}
$$

The aim of the low-complexity detector is to accurately estimate $l_{2 t-1}, l_{2 t}, s_{2 t-1}$ and $s_{2 t-1}$. Specifically, according to (20)-(24), the received signal $\mathbf{y}_{2 t-1}$ and $\mathbf{y}_{2 t}$ in $\mathbf{Y}_{t} \in$ $\mathbb{C}^{N_{r} \times 2}$ for this case can be represented as Eq. (50).

Hence, the signal matrix $\left[\mathbf{y}_{2 t-1}, \mathbf{y}_{2 t}^{*}\right]$ can be expressed as

$$
\left[\begin{array}{l}
\mathbf{y}_{2 t-1} \\
\mathbf{y}_{2 t}^{*}
\end{array}\right]=\mathbf{W}_{t}\left[\begin{array}{l}
s_{2 t-1} \\
s_{2 t}^{*}
\end{array}\right]+\left[\begin{array}{l}
\mathbf{n}_{2 t-1} \\
\mathbf{n}_{2 t}^{*}
\end{array}\right]
$$

where $\mathbf{W}_{t}$ can be expressed as Eq. (52), which is on the top of next page. For the index group $\Lambda_{p}=\left\{1,2, \ldots, N, 1 e^{j \theta}, \ldots,(L-N) e^{j \theta}\right\}$, there are a total number of $L^{2}$ index combinations $\mathbb{I}_{p}$ expressed as $\mathbb{I}_{p}=$ $\{\underbrace{(1,1)}_{I_{1}=(1,1)}, \ldots, \underbrace{\left((L-N) e^{j \theta},(L-N) e^{j \theta}\right)}_{I_{L}=(L, L)}\}$. For each mapping index combination $\left(l_{2 t-1}, l_{2 t}\right) \in \mathbb{I}_{m} m \in\left(1, L^{2}\right)$, we can obtain the corresponding channel matrix $\mathbf{W}_{t}^{m}$ according to (52), and the resultant symbol vector can be estimated as

$$
\hat{\mathbf{s}}_{t}^{m}=\left[\begin{array}{l}
\hat{s}_{2 t-1} \\
\hat{s}_{2 t}^{*}
\end{array}\right]=\mathbb{D}\left(\left(\mathbf{W}_{t}^{m}\right)^{\dagger}\left[\begin{array}{l}
\mathbf{y}_{2 t-1} \\
\mathbf{y}_{2 t}^{*}
\end{array}\right]\right) .
$$

After formulating $L^{2}$ symbol vectors as $\left(I_{1}, \hat{\mathbf{s}}_{t}^{1}\right), \ldots,\left(I_{N^{2}}, \hat{\mathbf{s}}_{t}^{L^{2}}\right)$, the optimal one can be estimated as

$$
\hat{m}=\arg \min _{m \in\left(1, L^{2}\right)}\left\|\left[\begin{array}{l}
\mathbf{y}_{2 t-1} \\
\mathbf{y}_{2 t}^{*}
\end{array}\right]-\mathbf{W}_{t}^{m} \hat{s}_{t}^{m}\right\|_{F}^{2} .
$$

Consequently, the final estimated signal can be expressed as $\left(I_{\hat{m}}, \hat{\mathbf{s}}_{t}^{\hat{m}}\right)$, which can be directly demodulated into the original information bits.

\section{E. Complexity Analysis of the Twin-RF ESM-ATA Detec- tors}

In this section, the complexity orders of both the ML and of the low-complexity suboptimal ESM-ATA detectors are analyzed in terms of the numbers of real-valued multiplications and additions. According to (41), the complexity order of the ML detector is expressed as

$$
C_{\mathrm{ML}}^{\mathrm{ESM}}=\left(16 N_{r} N_{t}+8 N_{r}-2\right)(L M)^{2},
$$

since $\left\|\mathbf{Y}_{t}-\mathbf{H X}_{t}\right\|_{F}^{2}$ requires $\left(16 N_{r} N_{t}+8 N_{r}-2\right)$ Flops, and this operation is computed $(L M)^{2}$ times.

By contrast, according to Eqs. (47)-(53), the lowcomplexity sub-optimal detector's complexity becomes

$$
C_{\mathrm{LC}}^{\mathrm{ESM}}=\left(76 N_{r}+64\right) L^{2}
$$

since

1) $\left(\mathbf{W}_{t}^{m}\right)^{\dagger}\left[\begin{array}{l}\mathbf{y}_{2 t-1} \\ \mathbf{y}_{2 t}^{*}\end{array}\right]$ imposes $32 N_{r}+4 N r+64$ Flops [23].

2) Eq. (47) or (53) represents $32 N_{r}+8 N r$ Flops.

\section{BER Performance Analysis of the Proposed SM-ATA AND ESM-ATA SCHEMES}

\section{A. BER Performance Analysis of Single-RF SM-ATA}

Let us denote the transmit and receive signal of SMATA by $\mathbf{x}_{i}$ and $\mathbf{x}_{j}$, respectively. Then the ABEP upper bound is given by:

$$
P_{b}=\frac{1}{B 2^{B}} \sum_{i=1}^{2^{B}} \sum_{j=1, j \neq i}^{2^{B}} d\left(\mathbf{x}_{i}, \mathbf{x}_{j}\right) P\left(\mathbf{x}_{i} \rightarrow \mathbf{x}_{j}\right),
$$




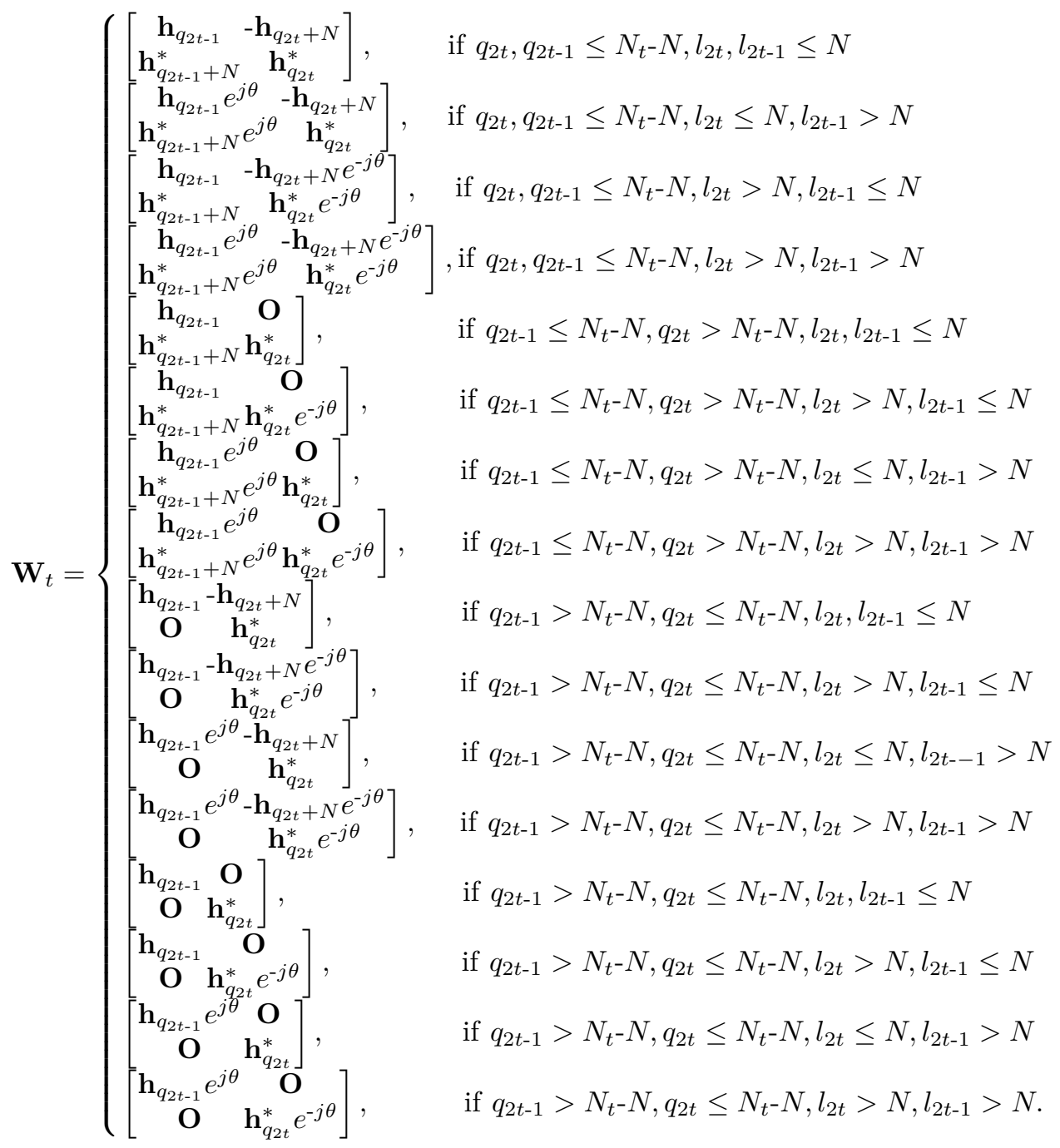

where $P\left(\mathbf{x}_{i} \rightarrow \mathbf{x}_{j}\right)$ denotes the Pairwise Error Probability (PEP) and $d\left(\mathbf{x}_{i}, \mathbf{x}_{j}\right)$ is the number of bit errors associated with the corresponding PEP event. Based on [23], the PEP is expressed as

$$
P\left(\mathbf{x}_{i} \rightarrow \mathbf{x}_{j} \mid \mathbf{H}\right)=Q\left(\sqrt{\frac{\left\|\mathbf{H}\left(\mathbf{x}_{i}-\mathbf{x}_{j}\right)\right\|^{2}}{2 \sigma^{2}}}\right)=Q(\sqrt{\zeta})
$$

where $Q(x)=(1 / \sqrt{2 \pi}) \int_{x}^{\infty} e^{-t^{2} / 2} d_{t}$ and $\zeta=\frac{\left\|\mathbf{H}\left(\mathbf{x}_{i}-\mathbf{x}_{j}\right)\right\|^{2}}{2 \sigma^{2}}$. The average PEP can be written as [23]

$$
\bar{P}\left(\mathbf{x}_{i} \rightarrow \mathbf{x}_{j}\right)=\gamma(\bar{\zeta}) \sum_{k=0}^{N_{r}-1}\left(\begin{array}{c}
N_{r}-1+k \\
k
\end{array}\right)[1-\gamma(\bar{\zeta})]^{k}
$$

where we have $\gamma(\bar{\zeta})=\frac{1}{2}\left(1-\sqrt{\frac{\bar{\zeta} / 2}{1+\bar{\zeta} / 2}}\right)$ and $\bar{\zeta}$ is the mean value of $\zeta$ with $N_{r}=1$.

Assuming that $l_{i}$ and $l_{j}$ are the mapping indices of $\mathbf{x}_{i}$ and $\mathbf{x}_{j}, q_{i}$ and $q_{j}$ are the corresponding activated TA indices of $\mathbf{x}_{i}$ and $\mathbf{x}_{j}, s_{i}$ and $s_{j}$ are the modulated $M$ ary APM symbols of of $\mathbf{x}_{i}$ and $\mathbf{x}_{j}$, we have the value of
$\bar{\zeta}_{\mathrm{SM}-\mathrm{ATA}}=E\left(\frac{\left\|\mathbf{H}\left(\mathbf{x}_{i}-\mathbf{x}_{j}\right)\right\|^{2}}{2 \sigma^{2}} \mid N_{r}=1\right)$ is expressed as

$$
\bar{\zeta}_{\mathrm{SM}-\mathrm{ATA}}=\left\{\begin{array}{ll}
\frac{\left|s_{i}-s_{j}\right|^{2}}{2 \sigma^{2}}, & \text { if } q_{i}=q_{j}, l_{i} \leq N_{t}, l_{j} \leq N_{t} \\
\frac{\left|s_{i} e^{j \theta}-s_{j}\right|^{2}}{2 \sigma^{2}}, & \text { if } q_{i}=q_{j}, \text { if } l_{i}>N_{t}, l_{j} \leq N_{t} \\
\frac{\left|s_{i}-s_{j} e^{j \theta}\right|^{2}}{2 \sigma^{2}}, & \text { if } q_{i}=q_{j}, l_{i} \leq N_{t}, l_{j}>N_{t} \\
\frac{\left|s_{i} e^{j \theta}-s_{j} e^{j \theta}\right|^{2}}{2 \sigma^{2}}, & \text { if } q_{i}=q_{j}, l_{i}>N_{t}, l_{j}>N_{t} \\
\frac{\left|s_{i}\right|^{2}+\left|s_{j}\right|^{2}}{2 \sigma^{2}}, & \text { if } q_{i} \neq q_{j}
\end{array} .\right.
$$

Accordingly, the ABEP of the proposed SM-ATA scheme can be expressed based on Eqs.(56), (58) and (59).

\section{B. BER Performance Analysis of Twin-RF ESM-ATA}

The ABEP upper bound is given as

$$
P_{b}=\frac{1}{2 B 2^{2 B}} \sum_{i=1}^{2^{2 B}} \sum_{j=1, j \neq i}^{2^{2 B}} d\left(\mathbf{X}_{i}, \mathbf{X}_{j}\right) P\left(\mathbf{X}_{i} \rightarrow \mathbf{X}_{j}\right),
$$

where $P\left(\mathbf{X}_{i} \rightarrow \mathbf{X}_{j}\right)$ denotes the PEP and $d\left(\mathbf{X}_{i}, \mathbf{X}_{j}\right)$ is the number of bit errors associated with the corresponding PEP event. Moreover, $P\left(\mathbf{X}_{i} \rightarrow \mathbf{X}_{j}\right)$ can be expressed as

$$
P_{e}\left(\mathbf{X}_{i} \rightarrow \mathbf{X}_{j}\right)=Q\left(\sqrt{\frac{\left\|\mathbf{H}\left(\mathbf{X}_{j}-\mathbf{X}_{i}\right)\right\|_{F}^{2}}{2 \sigma^{2}}}\right) .
$$




$$
P_{e}\left(\mathbf{X}_{i} \rightarrow \mathbf{X}_{j}\right)=\frac{1}{\pi} \int_{0}^{\pi / 2}\left(\frac{1}{1+\frac{\lambda_{i, j, 1}}{4 \sigma^{2} \sin ^{2} \phi}}\right)^{N_{r}} \times\left(\frac{1}{1+\frac{\lambda_{i, j, 2}}{4 \sigma^{2} \sin ^{2} \phi}}\right)^{N_{r}} \times \cdots \times\left(\frac{1}{1+\frac{\lambda_{i, j, \kappa i, j}}{4 \sigma^{2} \sin ^{2} \phi}}\right)^{N_{r}} d \phi
$$

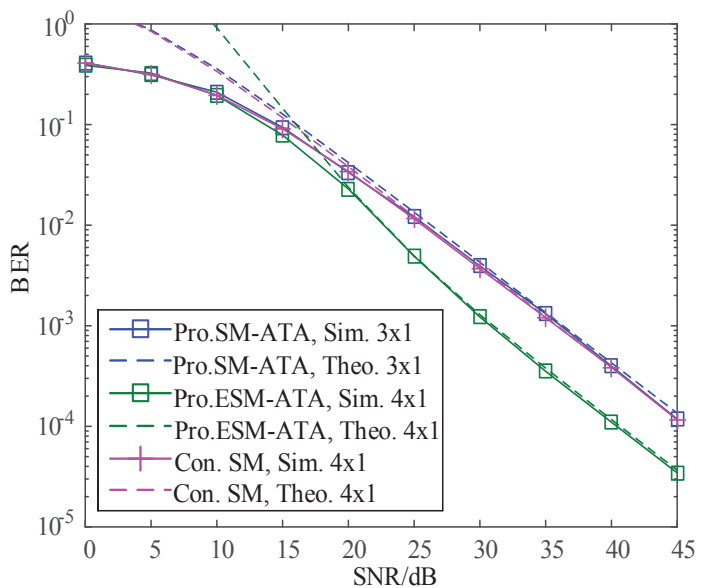

Fig. 4. Simulation and theoretical performance comparison of the QPSK-aided SM-ATA for $N_{t}=3, N_{r}=1$, of the QPSK-aided ESMATA for $N_{t}=4, N_{r}=1$ and of the QPSK-aided conventional SM scheme for $N_{t}=4, N_{r}=1$ at $4 \mathrm{bits} / \mathrm{s} / \mathrm{Hz}$. The theoretical results are computed by Eq. (57) or Eq. (61).

Considering that $Q(x)=\frac{1}{\pi} \int_{0}^{\pi / 2} \exp \left(-x^{2} / 2 \sin ^{2} \theta\right) d \theta$, Eq. (61) can be rewritten as

$$
P_{e}\left(\mathbf{X}_{i} \rightarrow \mathbf{X}_{j}\right)=E\left\{\frac{1}{\pi} \int_{0}^{\pi / 2} \exp \left(-\frac{\left\|\mathbf{H}\left(\mathbf{X}_{j}-\mathbf{X}_{i}\right)\right\|_{F}^{2}}{4 \sigma^{2} \sin ^{2} \theta}\right) d \theta\right\}
$$

Averaging (63) over the channel matrix $\mathbf{H}$ and using the moment generating function (MGF) approach [30], the PEP is obtained as (64), where $\kappa_{i, j}$ is the rank of the distance matrix $\mathbf{D}_{i, j}=\left(\mathbf{X}_{i}-\mathbf{X}_{j}\right)\left(\mathbf{X}_{i}-\mathbf{X}_{j}\right)^{H}$, and $\lambda_{i, j, 1}, \cdots, \lambda_{i, j, \kappa_{i, j}}$ are the non-zero eigenvalues of $\mathbf{D}_{i, j}$.

\section{Simulation Results}

In this subsection, the performances of the proposed SM-ATA and ESM-ATA schemes are presented and compared in Figs. 4 and 12. In all the simulation results, perfect channel state information is assumed and the values of the specific $\theta$ are selected based on Eq. (8). Specifically, Fig. 4 shows the theoretical and simulation performances of our QPSK-aided SM-ATA scheme for $N_{t}=3$ and $N_{r}=1$ and those of our QPSK-aided ESMATA scheme for $N_{t}=4$ and $N_{r}=1$ at 4 bits $/ \mathrm{s} / \mathrm{Hz}$. Moreover, the theoretical and simulation performances of the QPSK-aided conventional SM scheme associated with $N_{t}=4$ and $N_{r}=1$ are added as benchmarkers. As seen from Fig. 4, the upper bound derived becomes very tight upon increasing the SNR values for the proposed SM-ATA and ESM-ATA schemes, which is helpful for evaluating the BER performances of the proposed SM-ATA and ESMATA schemes. Moreover, it is shown that the proposed

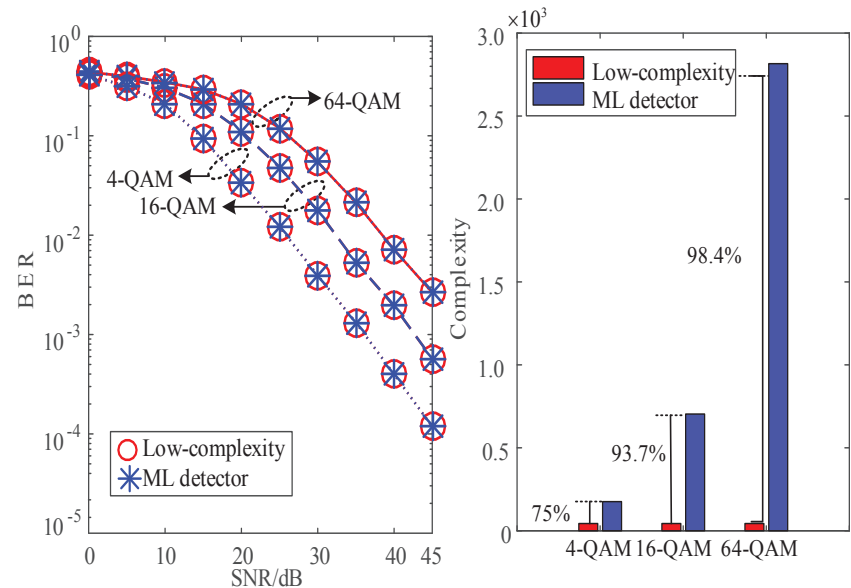

Fig. 5. Performance and complexity comparisons of the ML detector and of the low-complexity ML detector for the 4-QAM-aided, 16QAM-aided and 64-QAM-aided SM-ATA schemes having $N_{t}=3$ and $N_{r}=1$ at 4 bits $/ \mathrm{s} / \mathrm{Hz}, 6 \mathrm{bits} / \mathrm{s} / \mathrm{Hz}$ and $8 \mathrm{bits} / \mathrm{s} / \mathrm{Hz}$.
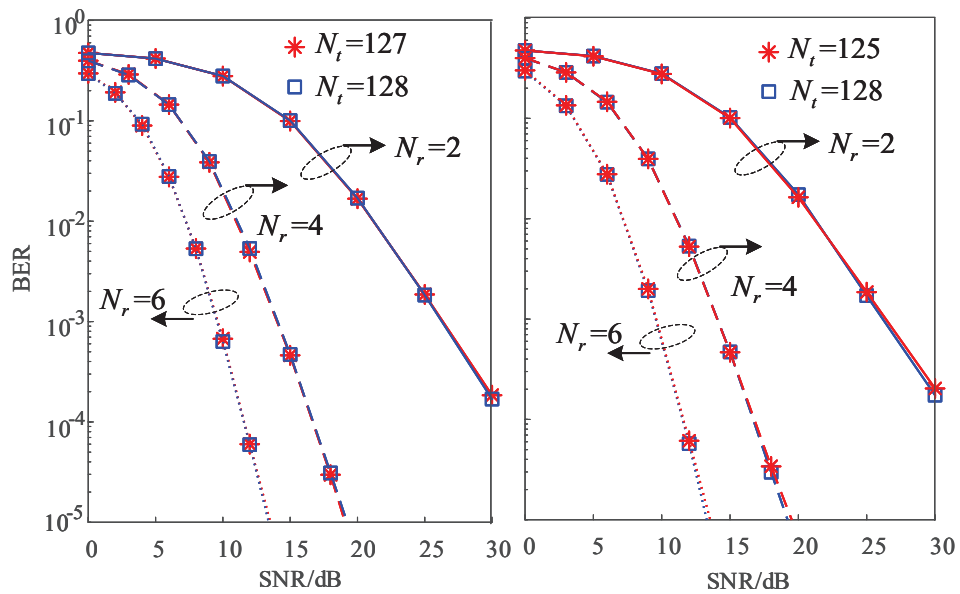

Fig. 6. Performance of the proposed SM-ATA schemes having $N_{t}=$ 127 and $N_{t}=125$ at 9 bits $/ \mathrm{s} / \mathrm{Hz}$. The corresponding conventional SM schemes having $N_{t}=128$ were added as benchmarkers.

SM-ATA scheme approaches the performance of the conventional SM scheme, despite having less TAs, while the proposed ESM-ATA scheme is capable of providing an approximately $5 \mathrm{~dB}$ gain over the conventional SM scheme.

\section{A. Performance comparisons of Single-RF SM-ATA scheme}

Fig. 5 compares the BER performance and complexity of both the proposed low-complexity detector and of the ML detector for the 4-QAM-aided, 16-QAM-aided, and 64-QAM-aided SM-ATA schemes associated with $N_{t}=4$ and $N_{r}=1$. It becomes evident that the proposed lowcomplexity detector imposes only $10 \%$ of the ML detector's complexity at the same performance. The complexity 
advantage becomes more dominant for high modulation orders.

Fig. 6 characterizes the BER performance of the SMATA scheme having $N_{t}=127$ and $N_{t}=125$ at 9 bits/s/Hz in conjunction with different number of receiver antennas. The conventional SM schemes associated with $N_{t}=128$ having the same transmission rate are also added as benchmarkers. For the case of $N_{t}=127$, the extended index group is $\Lambda_{p}=\left\{1,2, \ldots, 127, q_{1} e^{j \theta}\right\}$, while we have $\Lambda_{p}=\left\{1,2, \ldots, 125, q_{1} e^{j \theta}, q_{2} e^{j \theta}, q_{3} e^{j \theta}\right\}$ for the case of $N_{t}=125$. Moreover, the repeated indices of $q_{1}, q_{2}, q_{3}$ are selected randomly from the conventional index group of $\Lambda=\left\{1,2, \ldots, N_{t}\right\}$. Observe from Fig. 6 that the proposed SM-ATA schemes associated with $N_{t}=127$ and $N_{t}=125$ are capable of achieving nearly the same performance as the conventional SM scheme associated with $N_{t}=128$ at the same throughput for the case of $N_{r}=2, N_{r}=4$ and $N_{r}=6$.

Compared to the VTM scheme of [19] and to the 3D based SM scheme of [20], the main difference lies in the complexity of bit-to-symbol mapping. For the VTM scheme of [19], the complexity order of the bit-to-symbol mapping is $O\left(N_{t}\right)+C_{b i t 2 d e c}$, where $O\left(N_{t}\right)$ is the complexity order of bit-to-antenna-index mapping and $C_{b i t 2 d e c}$ is the complexity of transforming a block of bits into a decimal format. The complexity order of the bit-to-symbol mapping in [20] is $2^{M}+C_{2^{n}}^{2}+C_{2^{n}+N_{t}-1, N_{t}-1}+O\left(2^{n}\right)+C_{b i t 2 d e c}$, where $2^{M}+C_{2^{n}}^{2}+C_{2^{n}+N_{t}-1, N_{t}-1}$ is the complexity order of the constellation design, $O\left(2^{n}\right)$ is the complexity order of the bit-to-antenna-index mapping. The complexity order of the bit-to-symbol mapping of the proposed scheme is $C_{b i t 2 d e c}$. Hence, the proposed scheme imposes a reduced bit-to-symbol mapping complexity in massive MIMO setups.

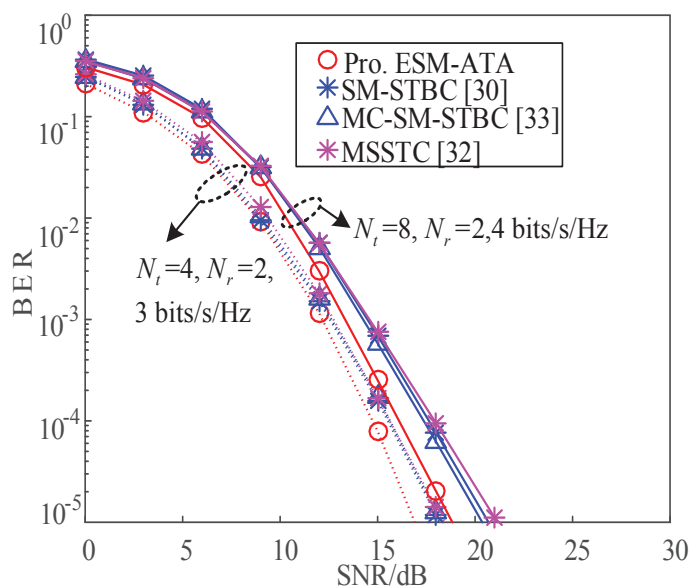

Fig. 7. Performance comparison between the proposed ESM-ATA scheme and the conventional STBC-SM schemes at $3 \mathrm{bits} / \mathrm{s} / \mathrm{Hz}$ and $4 \mathrm{bits} / \mathrm{s} / \mathrm{Hz}$.

\section{B. Performance comparisons of Twin-RF ESM-ATA Sys- tems}

Figs. 7-9 compare the BER performance of the proposed ESM-ATA schemes to that of the existing twin-RF

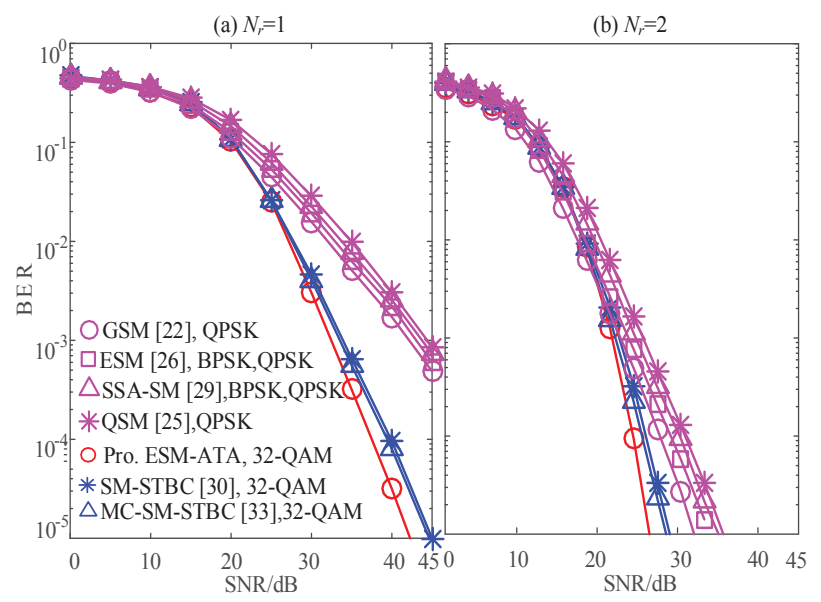

Fig. 8. Performance comparison between the proposed ESM-ATA scheme and the existing twin-RF based SM schemes having $N_{t}=4$ at 6 bits $/ \mathrm{s} / \mathrm{Hz}$; a) $N_{r}=1$; b) $N_{r}=2$.

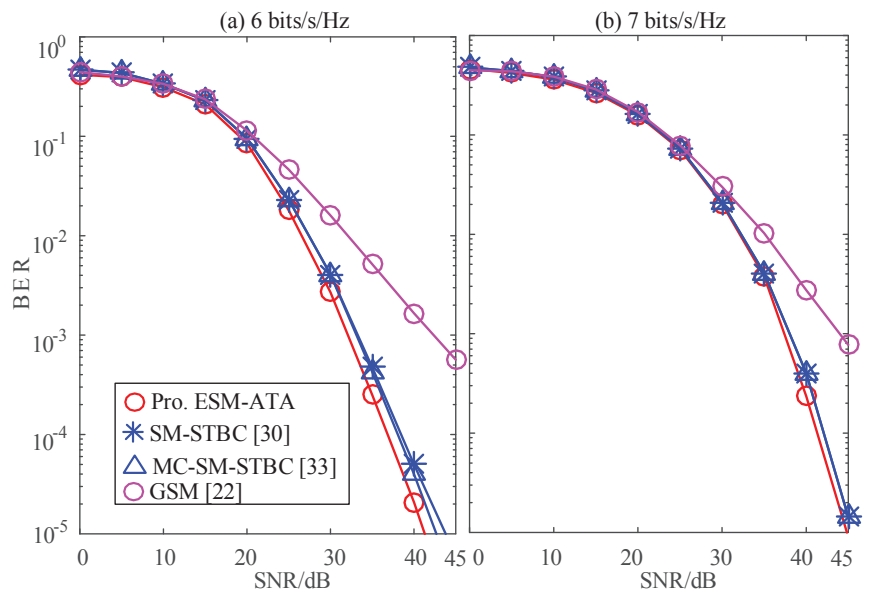

Fig. 9. Performance comparison between the proposed ESM-ATA scheme and the existing twin-RF based SM schemes having $N_{t}=6$ at $5 \mathrm{bits} / \mathrm{s} / \mathrm{Hz}, 6 \mathrm{bits} / \mathrm{s} / \mathrm{Hz}, 7 \mathrm{bits} / \mathrm{s} / \mathrm{Hz}$.

schemes in conjunction with different antenna configurations. ML detectors are employed for the simulation results of Figs. 7-9. Specifically, Fig. 7 compares the performance of the proposed ESM-ATA schemes to that of the spacetime coding based SM schemes having $N_{t}=4 N_{r}=2$ and $N_{t}=8 N_{r}=2$. QPSK is employed in the proposed ESMATA, STBC-SM [30] and MC-STBC-SM [33] schemes, while BPSK is employed in the MSSTC scheme [32]. Observe from Fig. 7 that the ESM-ATA scheme associated with $N_{t}=4, N=2, N_{r}=2$ and $N_{t}=8, N=2, N_{r}=2$ outperforms the classic SM-STBC schemes by about $1 \mathrm{~dB}$ and $2 \mathrm{~dB}$ at $\mathrm{BER}=10^{-5}$, respectively.

Fig. 8 compares the performances of the proposed ESMATA schemes to that of the existing twin-RF SM schemes having $N_{t}=4$ at 6 bits $/ \mathrm{s} / \mathrm{Hz}$. 32-QAM is employed by the ESM-ATA, STBC-SM and MC-STBC-SM schemes. Furthermore, QPSK is employed in the GSM and QSM schemes, while mixed BPSK and QPSK are employed in the ESM and SSA-SM schemes. For the case of $N_{t}=$ $4, N_{r}=2$, the ESM-ATA scheme associated with $N=2$ 
outperforms the STBC-SM, the MC-STBC-SM, the GSM, the ESM, the SSA-SM, and the QSM schemes by around $2 \mathrm{~dB}, 2.5 \mathrm{~dB}, 5 \mathrm{~dB}, 7 \mathrm{~dB}, 8 \mathrm{~dB}$ and $9 \mathrm{~dB}$ at $\mathrm{BER}=10^{-5}$. The performance advantages become more dominant for the case of $N_{t}=4, N_{r}=1$.

Fig. 9 compares the performance of the proposed ESMATA scheme to that of the space-time coding based twin SM schemes and to the multiplexing based SM schemes having $N_{t}=6 N_{r}=1$ at 6 bits $/ \mathrm{s} / \mathrm{Hz}$ and 7 bits $/ \mathrm{s} / \mathrm{Hz}$. 16-QAM, 32-PSK and $N=3$ are employed for ESM-ATA scheme to achieve $6 \mathrm{bits} / \mathrm{s} / \mathrm{Hz}$ and $7 \mathrm{bits} / \mathrm{s} / \mathrm{Hz}$. For the case of $N_{t}=6$, only the GSM scheme is suitable for the twin-RF chain scenario at the transmitter. Mixed BPSKQPSK and QPSK are employed for the GSM schemes to achieve 6 bits/s/Hz and 7 bits/s/Hz. Observe from Fig. 9 that the proposed ESM-ATA schemes provide considerable performance gains over the GSM schemes and offer a similar performance as the STBC-SM and MC-STBC-SM schemes.

Fig. 10 compares both the BER performance and complexity of the proposed low-complexity detector and of the ML detector for the 4-QAM-aided ESM-ATA schemes in conjunction with different number of receiver antennas at $4 \mathrm{bits} / \mathrm{s} / \mathrm{Hz}$. It is shown that the ESM-ATA associated with the proposed low-complexity detector is capable of achieving about $90 \%$ complexity reduction over its ML counterpart at the cost of around $1 \mathrm{~dB}$ performance loss for the case of $N_{r}=1$. Moreover when the value of $N_{r}$ increases, the ESM-ATA scheme using the proposed lowcomplexity detector offers nearly the same performance as its ML counterpart, despite its $90 \%$ complexity reduction.

Fig. 11 presents the performances of both the ML and of the Low-complexity (LC) detector based ESM-ATA schemes having $N_{t}=32, N=16, N_{r}=2$ and $N_{t}=30$, $N=15, N_{r}=2$. QPSK and 16-QAM are employed for the ESM-ATA schemes to achieve throughputs of 6 bits $/ \mathrm{s} / \mathrm{Hz}$ and $8 \mathrm{bits} / \mathrm{s} / \mathrm{Hz}$. The performances of the ML detector based STBC-SM [30], of the Alamouti [36] and of the GSM schemes having the same transmission rates were also added for comparisons. Our simulation results have shown that the proposed ESM-ATA scheme associated with LC detector is capable of approaching the performance of its ML counterpart for these setups. The QPSK-aided ESM-ATA schemes having $N_{t}=32, N=16, N_{r}=2$ and $N_{t}=30, N=15 N_{r}=2$ offer nearly the same performance and outperform the STBC-SM scheme [30] and the Alamouti [36] scheme by $2 \mathrm{~dB}$ and $6 \mathrm{~dB}$ at $\mathrm{BER}=10^{-5}$, respectively. The 16-QAM-aided ESM-ATA scheme having $N_{t}=32, N=16, N_{r}=2$ outperforms the STBC-SM scheme [30] and the GSM scheme [22] by $2 \mathrm{~dB}$ and $10 \mathrm{~dB}$ at $\mathrm{BER}=10^{-5}$, respectively.

Finally, Fig. 12 presents the performances of the LC detector based ESM-ATA schemes advocated, which have $N_{t}=128$ and $N_{t}=256$ associated with $N_{r}=2$ and $N_{r}=4$ at 8 bits $/ \mathrm{s} / \mathrm{Hz}$. In these setups, the existing STBCSM scheme of $[30]$ becomes impractical due to the high complexity of its bit-to-symbol mapping. The performance of the Alamouti scheme associated with $8 \mathrm{bits} / \mathrm{s} / \mathrm{Hz}$ was
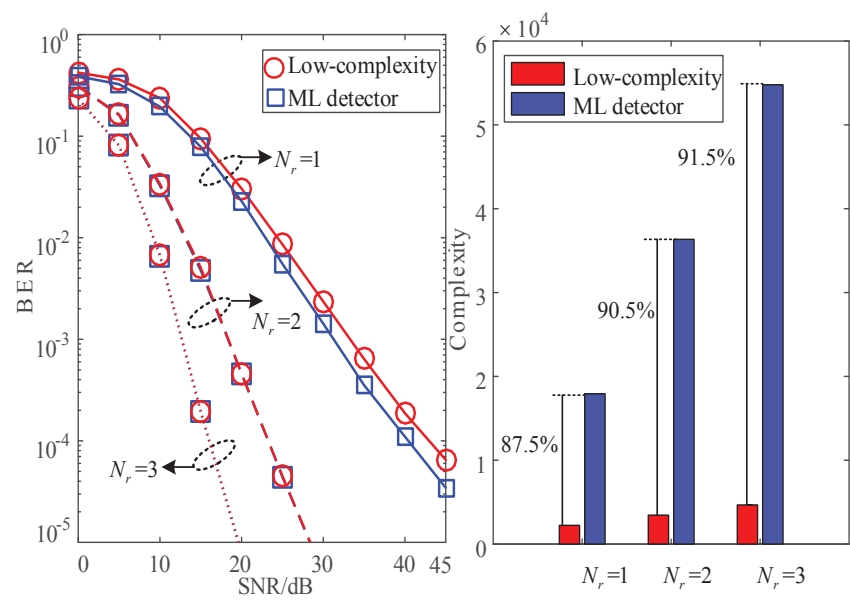

Fig. 10. Performance and complexity comparisons of the ML detector and of the low-complexity ML detector for the 4-QAM-aided ESMATA scheme having $N_{t}=4$ and $N=3$ for different values of $N_{r}$ at $4 \mathrm{bits} / \mathrm{s} / \mathrm{Hz}$.
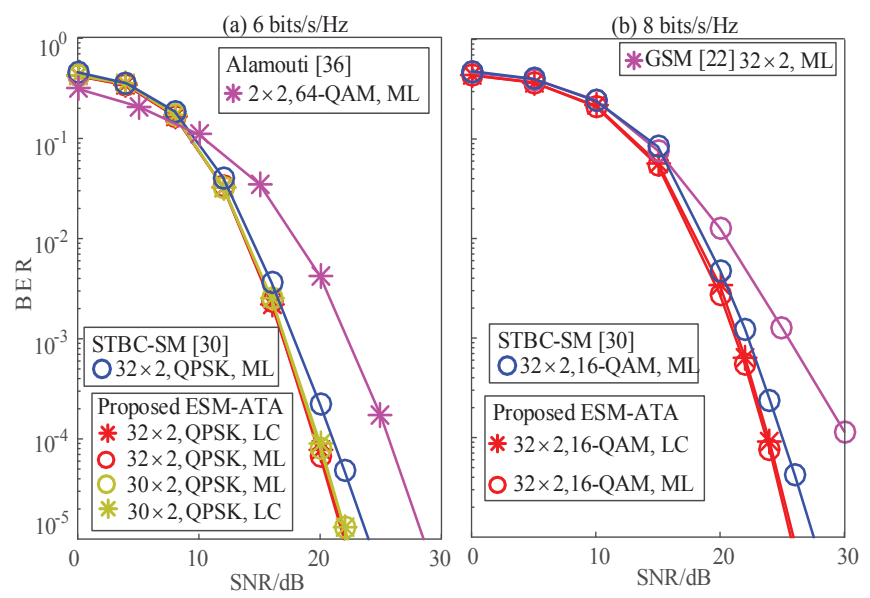

Fig. 11. Performance comparisons of the proposed ESM-ATA scheme to the existing twin-RF schemes having a throughput of 6 bits $/ \mathrm{s} / \mathrm{Hz}$ and $8 \mathrm{bits} / \mathrm{s} / \mathrm{Hz}$.

also added as a benchmarker. Observe from Fig. 12 that the proposed scheme having two-RF chains is capable of attaining in excess of $15 \mathrm{~dB}$ performance gain over the conventional Alamouti scheme for both $N_{r}=2$ and for $N_{r}=4$.

\section{Conclusions}

In this paper, a novel single RF-aided SM scheme is proposed for an arbitrary number of TAs. Based on the proposed SM-ATA scheme, a twin-RF ESM-ATA is also proposed for an arbitrary number of TAs. Low-complexity detectors are designed for both the SM-ATA and ESMATA schemes, which are capable of achieving $90 \%$ complexity reduction at a negligible performance loss. Both our simulation and theoretical results have demonstrated that the proposed SM-ATA is capable of approaching the same performance as the conventional SM system at a reduced number of TAs, while the proposed ESM-ATA scheme is capable of providing considerable performance 


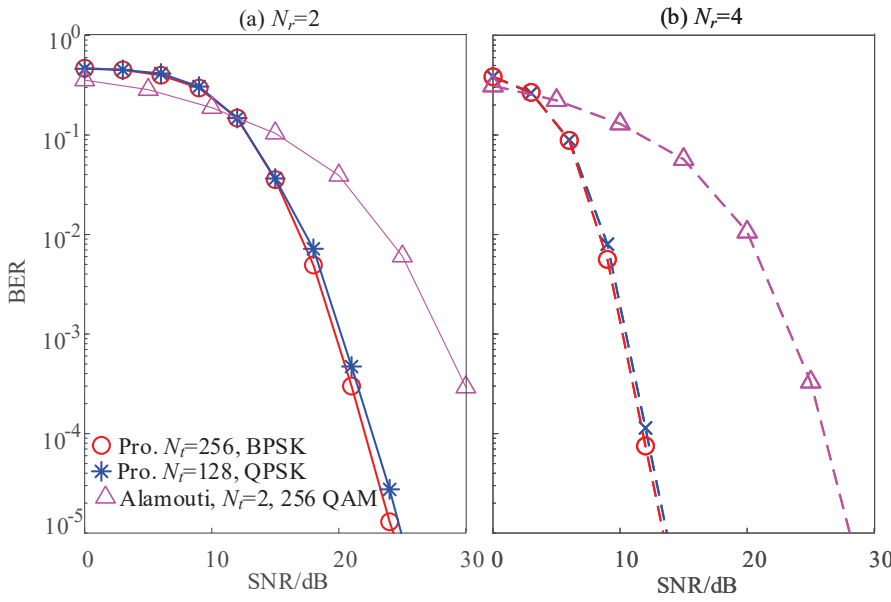

Fig. 12. Performance comparisons of the proposed ESM-ATA scheme to the existing twin-RF schemes having a throughput of $8 \mathrm{bits} / \mathrm{s} / \mathrm{Hz}$.

gains over the conventional twin-RF based SM system at the same number of TAs. Moreover, the proposed single-RF and twin-RF schemes are more attractive for employment in massive MIMO downlink communications.

\section{REFERENCES}

[1] R. Mesleh, H. Haas, S. Sinanovic, C. W. Ahn, and S. Yun, "Spatial modulation," IEEE Trans. Veh. Technol., vol. 57, no. 4, pp. 2228-2241, Jul. 2008.

[2] M. Di Renzo, H. Haas, and P. M. Grant, "Spatial modulation for multiple-antenna wireless systems: A survey," IEEE Commun. Mag., vol. 49, no. 12, pp. 182-191, Dec. 2011.

[3] M. Di Renzo, H. Haas, A. Ghrayeb, S. Sugiura, and L. Hanzo, "Spatial modulation for generalized MIMO: challenges, opportunities and implementation," Proceedings of the IEEE, vol. 102, no. 1, pp. 56-103, Jan. 2014

[4] R. Rajashekar, K.V.S. Hari and L. Hanzo, "Reduced-complexity ML detection and capacity-optimized Training for spatial modulation systems," IEEE Trans. Commun., vol. 62, no. 1, pp. 112-125, Jan. 2014.

[5] P. Yang, M. Di Renzo, Y. Xiao, S. Q. Li and L. Hanzo,"Design guidelines for spatial modulation," IEEE Commun. Surveys Tuts., vol. 17, no. 1, pp. 6-26, First Quart. 2015.

[6] P. Yang, Y. Xiao, Y. L. Guan, K. V. S. Hari, A. Chockalingam, S. Sugiura, H. Haas, M. Di Renzo, C. Masouros, Z. Liu, L. Xiao, S. Li, and L. Hanzo, "Single-carrier spatial modulation: A promising design for large-scale broadband antenna systems," IEEE Commun. Surveys Tuts., vol. 18, no. 3, pp. 1687-1716, Feb. 2016.

[7] P. Liu, M. Di. Renzo and A. Springer, "Line-of-sight spatial modulation for indoor mmwave communication at $60 \mathrm{GHz}$," IEEE Trans. Wireless Commun., vol. 15, no. 11, pp. 7373-7389, Nov. 2016.

[8] L. He, J. Wang, J. Song, and L. Hanzo, "On the multi-user, multi-cell massive spatial modulation uplink: How many antennas for each user?,"IEEE Trans. Wireless Commun., vol. PP, no. 99, pp. 1-1, Dec. 2016

[9] P. Yang, Y. Xiao, B. Zhang, S. Q. Li, M. El-Hajjar, and L. Hanzo, "Star-QAM signaling constellations for spatial modulation," IEEE Trans. Veh. Technol., vol. 63, no. 8, pp. 3741-3749, Oct. 2014.

[10] P. Yang, Y. Xiao, L. Yin, Q. Tang, S. Q. Li, and L. Hanzo, "Hybrid bit-to-symbol mapping for spatial modulation," IEEE Trans. Veh. Technol., vol. 65, no. 7, pp. 5804-5810, July. 2015.
[11] M. Maleki, H. Reza Bahrami, A. Alizadeh, and N. H. Tran, "On the performance of spatial modulation: optimal constellation breakdown,"IEEE Trans. Commnun., vol. 62, no. 1, pp. 144157, Jan. 2014.

[12] P. Yang, Y. Xiao, Y. Yu, abd S. Q. Li, "Adaptive spatial modulation for wireliess MIMO transmission systems," IEEE Commun. Lett., vol. 15, no. 6, pp. 602-606, Jun. 2011.

[13] C. Masouros and L. Hanzo, "Constellation randomization achieves transmit diversity for single-RF spatial modulation,"IEEE Trans. Veh. Technol., vol. 65, no. 10, pp. 8101-8111, Oct. 2016.

[14] C. Masouros and L. Hanzo, "A scalable performance-complexity tradeoff for constellation randomization in spatial modulation," IEEE Trans. Veh. Technol., vol. 66, no. 3, pp. 2834-2838, Mar. 2017.

[15] C. Liu, M. Ma, Y. Yang, "Optimal spatial-domain design for spatial modulation capacity maximization,"IEEE Commun. Lett., vol. 20, no. 6, pp. 1092-1096, Jun. 2016.

[16] M. S. Veedu, C. R. Murthy, and L. Hanzo, "Single-RF spatial modulation relying on finite-rate phase-only feedback: Design and snalysis," IEEE Trans. Veh. Technol., vol. 65, no. 4, pp. 2016-2025, Apr. 2016.

[17] N. Serafimovski, M. Di Renzo, S. Sinanovic, R. Y. Mesleh and H. Haas, "Fractional bit encoded spatial modulation," IEEE Commun. Lett., vol. 14, no. 5, pp. 429-431, May. 2010.

[18] Y. Yang and S. Aissa, "Bit-padding information guided channel hopping,"IEEE Commun. Lett., vol. 15, no. 2, pp. 163-165, Feb. 2011.

[19] Y. Yang and S. Aissa, "Information guided channel hopping with an arbitrary number of transmit antennas,"IEEE Commun. Lett., vol. 16, no. 10, pp. 1552-1555, Oct. 2012.

[20] S. Guo, H. Zhang, S. Jin, and P. Zhang, "Spatial modulation via 3-D mapping,"IEEE Commun. Lett., vol. 20, no. 6, pp. 10961099,Jun. 2016.

[21] T. Handte, A. Muller, and J. Speidel, "BER analysis and optimization of generalized spatial modulation in correlated fading channels," in Proc. 2009 VTC Fall, Anchorage, AK, USA, Jan. 2010, pp. 1-5.

[22] A. Younis, N. Serafimovski, R. Mesleh, and H. Haas, "Generalised spatial modulation," in Proc. 2010 Signals, Syt. Comput., Pacific Grove, Nov. 2010, pp. 1498-1502.

[23] L. Xiao, P. Yang, Y. Xiao, S. Fan, M. Di. Renzo, W. Xiang, and $\mathrm{S}$. Li, "Efficient compressive sensing detectors for generalized spatial modulation systems," IEEE Trans. Veh. Technol., DOI: 10.1109/TVT.2016.2558205.

[24] J. Freudenberger and S. Shavgulidze, "Signal constellations based on eisenstein integers for generalized spatial modulation, IEEE Commun. Lett., vol. 21, no. 3, pp. 556-559, Mar. 2017.

[25] R. Mesleh, S. S. Ikki, and H. M. Aggoune, "Quadrature spatial modulation," IEEE Trans. Veh. Technol., vol. 64, no. 6, pp. 2738-2742, June. 2015.

[26] C.-C. Cheng, H. Sari, S. Sezginer and Y. Su, "Enhanced spatial modulation with multiple signal constellations,"IEEE Trans. Commun., vol. 63, no. 6, June 2015, pp. 2237-2248.

[27] C.-C. Cheng, H. Sari, S. Sezginer and Y. Su, "New signal designs for enhanced spatial modulation "IEEE Trans. Wireless Commun., vol. 15, no. 11, pp. 7766-7777, Nov. 2016.

[28] O. Osman, "Variable active antenna spatial modulation," IEEE IET Microwaves, Antennas Propagation vol. 9, no. 15, pp. 18161824 Oct. 2015. 2237-48.

[29] P. Koundinya, K. V. S. Hari, and L. Hanzo, "Joint design of the spatial and of the classic symbol alphabet improves single-RF spatial modulation,"IEEE Access, vol. 4, pp. 10246-10257. Aug. 2016.

[30] E. Basar, U. Aygolu, E. Panayirci and H. V. Poor, "Space-Time 
Block Coded Spatial Modulation," IEEE Trans. Commun., vol. 59, no. 3, pp. 823-832, Mar. 2011.

[31] X. Li and L. Wang, "High rate space-time block coded spatial modulation with cyclic structure," IEEE Commun. Lett., vol. 18, no. 4, pp. 532-535, Apr. 2014.

[32] C. Jeon and J. Lee, "Multi-strata space-time coded spatial modulation," IEEE Commun. Lett., vol. 19, no. 11, pp. 19451948, Nov. 2015.

[33] Y. Hua, G. Zhao, W. Zhao, and M. Jin, "Modified codewords design for spaceĺCtime block coded spatial modulation," IEEE Trans. IET Commun., vol. 11, no. 2, pp. 249-257, Jan. 2017.
[34] M. C. Park, B. G. Jo and D. S. Han, "Double space-time transmit diversity with spatial modulation," IEEE Electronic Lett., vol. 51, no. 25, pp. 2155-2156, Dec. 2015.

[35] M. Le, V. Ngo, H. Mai, X. Nam, and M. Di. Renzo, "Spatially modulated orthogonal space-time block codes with nonvanishing determinants," IEEE Trans. Commun., vol. 62, no. 1, pp. 85-99, Jan. 2014.

[36] S. M. Alamouti, "A simple transmit diversity technique for wire958 less communications," IEEE J. Sel. Areas Commun., vol. 16, no. 8, 959 pp. 1451-1458, Oct. 1998. 